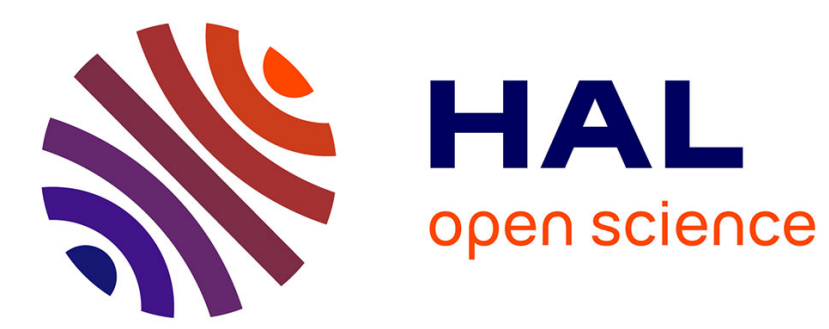

\title{
Occupation Times in Markov Processes
}

Bruno Sericola

\section{To cite this version:}

Bruno Sericola. Occupation Times in Markov Processes. [Research Report] RR-3806, INRIA. 1999. inria-00072852

\section{HAL Id: inria-00072852 \\ https://hal.inria.fr/inria-00072852}

Submitted on 24 May 2006

HAL is a multi-disciplinary open access archive for the deposit and dissemination of scientific research documents, whether they are published or not. The documents may come from teaching and research institutions in France or abroad, or from public or private research centers.
L'archive ouverte pluridisciplinaire HAL, est destinée au dépôt et à la diffusion de documents scientifiques de niveau recherche, publiés ou non, émanant des établissements d'enseignement et de recherche français ou étrangers, des laboratoires publics ou privés. 
INSTITUT NATIONAL DE RECHERCHE EN INFORMATIQUE ET EN AUTOMATIQUE

\title{
Occupation Times in Markov Processes
}

\author{
Bruno Sericola
}

$\mathbf{N}^{\circ} 3806$

Novembre 1999

THÈME 1 



\title{
RINRIA
}

\section{Occupation Times in Markov Processes}

\author{
Bruno Sericola * \\ Thème 1 - Réseaux et systèmes \\ Projet Armor \\ Rapport de recherche $\mathrm{n}^{\circ} 3806$ - Novembre 1999 - 41 pages
}

\begin{abstract}
We consider, in a homogeneous Markov process with finite state space, the occupation times that is, the times spent by the process in given subsets of the state space during a finite interval of time. We first derive the distribution of the occupation time of one subset and then we generalize this result to the joint distribution of occupation times of different subsets of the state space by the use of order statistics from the uniform distribution. Next, we consider the distribution of weighted sums of occupation times. We obtain the forward and backward equations describing the behavior of these weighted sums and we show how these equations lead to simple expressions of this distribution.
\end{abstract}

Key-words: Occupation times, Markov processes, order statistics, availability, performability.

(Résumé : tsvp)

* $\{$ Bruno.Sericola $\} @$ irisa.fr

Unité de recherche INRIA Rennes

IRISA, Campus universitaire de Beaulieu, 35042 RENNES Cedex (France)

Téléphone : 0299847100 - International : +33299847100

Télécopie : 0299847171 - International : +33299847171 


\section{Temps d'occupation dans les processus de Markov}

Résumé : On considère, dans un processus de Markov homogène à espace d'états fini, les temps d'occupation c'est-à-dire, les temps passés par le processus dans des sous-ensembles d'états donnés durant un intervalle de temps fini. On obtient dans un premier temps la distribution du temps d'occupation d'un sous-ensemble et on généralise ce résultat à la distribution jointe de temps d'occupation de différents sous-ensembles de l'espace d'états par l'utilisation de statistiques d'ordre de la loi uniforme. On considère ensuite la distribution de sommes pondérées de temps d'occupation. On obtient les équations avant et arrière décrivant le comportement de ces sommes pondérées et on montre comment ces équations conduisent à des expressions simples de cette distribution.

Mots-clé : Temps d'occupation, processus de Markov, statistiques d'ordre, disponibilité, performabilité. 


\section{Introduction}

Let $X=\left\{X_{u}, u \geq 0\right\}$ be a homogeneous Markov process with a finite state space $S$. The occupation time of a subset $U \subset S$ over the interval $[0, t)$ is defined by the random variable $W_{t}$ as

$$
W_{t}=\int_{0}^{t} 1_{\left\{X_{u} \in U\right\}} d u
$$

where $1_{\{c\}}=1$ if condition $c$ is true and 0 otherwise. This random variable received a considerable attention since it is also known as the interval availability in reliability and dependability theory. An expression of the distribution of $W_{t}$ has been obtained in [2] using order statistics from the uniform distribution over $[0, t)$. This expression is very interesting from a computational point of view and various methods have been developed to compute it even in the case of denumerable state spaces (see [2], [10], [8], [9] and the references therein).

In this paper, we first obtain the joint distribution of the random variables $\left(W_{t}, X_{t}\right)$ using the forward and backward equations associated to the uniformized Markov chain of the process $X$. We then generalize this result to the joint distribution of $W_{t}^{1}, \ldots, W_{t}^{m}, X_{t}$, where $W_{t}^{i}$ is the occupation time of a subset $B_{i}$ over the interval $[0, t)$. Finally, we consider a weighted sum of occupation times, that is the random variable $Y_{t}$ defined by

$$
Y_{t}=\int_{0}^{t} \rho\left(X_{u}\right) d u
$$

where for each $i \in S, \rho(i)$ is a non negative constant. The study of this random variable $Y_{t}$ is also known as the performability analysis in the reliability and dependability theory (see [3], [5] and the references therein). We derive here the backward and forward equations describing the behavior of the joint distribution of $\left(Y_{t}, X_{t}\right)$. These equations, which are partial differential equations, are then solved and we show that they lead to simple expressions of the joint distribution of $\left(Y_{t}, X_{t}\right)$.

The rest of paper is organized as follows. In the next section, we consider the joint distribution of order statistics from the uniform distribution and the joint conditional distribution of the jumps in a Poisson process and we show how they are related. In section 3 , we consider the case $m=1$. We obtain the distribution of occupation time for a discrete time Markov chain. This distribution combined with the results of section 2 lead to simple expressions of the joint distribution of the couple $\left(W_{t}, X_{t}\right)$. The results of section 3 are then generalized in section 4 to the case where $m>1$. Finally, section 5 deals with the distribution of the couple $\left(Y_{t}, X_{t}\right)$.

$\mathrm{RR} \mathrm{n}^{\circ} 3806$ 


\section{Order Statistics}

\subsection{The Uniform Distribution}

In this subsection, we consider order statistics from the uniform distribution over $[0, t)$, where $t$ is a fixed positive real number. More formally, let $X_{1}, X_{2}, \ldots, X_{n}$ be $n$ i.i.d. random variables with common distribution the uniform distribution over $[0, t)$. We thus have, for all $i=1, \ldots, n$ and $x \in \mathbb{R}$,

$$
\mathbb{P}\left\{X_{i} \leq x\right\}=\left\{\begin{array}{lll}
0 & \text { if } & x \leq 0 \\
x / t & \text { if } & x \in(0, t) \\
1 & \text { if } & x \geq t
\end{array}\right.
$$

If the random variables $X_{1}, X_{2}, \ldots, X_{n}$ are rearranged in ascending order of magnitude and then written as

$$
X_{(1)} \leq X_{(2)} \leq \cdots \leq X_{(n)}
$$

we call $X_{(i)}$ the $i$ th order statistic, $i=1,2, \ldots, n$. Because of the inequality relations among them the $X_{(i)}$ are necessarily dependent random variables.

Let $F_{r}(x)$ be the distribution of $X_{(r)}$. We then have, for $x \in(0, t)$,

$$
\begin{aligned}
F_{r}(x) & =\mathbb{P}\left\{X_{(r)} \leq x\right\} \\
& =\mathbb{P}\left\{\text { at least } r \text { of the } X_{k} \text { are less than or equal to } x\right\} \\
& =\sum_{i=r}^{n}\left(\begin{array}{c}
n \\
i
\end{array}\right)\left(\frac{x}{t}\right)^{i}\left(1-\frac{x}{t}\right)^{n-i}
\end{aligned}
$$

The density $f_{r}(x)$ of $X_{(r)}$ is thus given, for $x \in(0, t)$, by

$$
f_{r}(x)=\frac{n !}{(r-1) !(n-r) !} \frac{1}{t}\left(\frac{x}{t}\right)^{r}\left(1-\frac{x}{t}\right)^{n-r} .
$$

It is shown in [1] that the joint density function of $X_{\left(r_{1}\right)}, X_{\left(r_{2}\right)}, \ldots, X_{\left(r_{k}\right)}$, for $1 \leq k \leq n$, $1 \leq r_{1}<r_{2}<\cdots<r_{k} \leq n$ and $x_{1} \leq x_{2} \leq \cdots \leq x_{k}\left(x_{i} \in(0, t)\right)$ is

$$
\begin{aligned}
& f_{r_{1}, r_{2}, \ldots, r_{k}}\left(x_{1}, x_{2}, \ldots, x_{k}\right)= \\
& \quad \frac{n !\left(\frac{1}{t}\right)^{k}\left(\frac{x_{1}}{t}\right)^{r_{1}-1}\left(\frac{x_{2}-x_{1}}{t}\right)^{r_{2}-r_{1}-1} \cdots\left(\frac{x_{k}-x_{k-1}}{t}\right)^{r_{k}-r_{k-1}-1}\left(1-\frac{x_{k}}{t}\right)^{n-r_{k}}}{\left(r_{1}-1\right) !\left(r_{2}-r_{1}-1\right) ! \cdots\left(r_{k}-r_{k-1}-1\right) !\left(n-r_{k}\right) !}
\end{aligned}
$$


The joint density function of $X_{\left(l_{1}\right)}, X_{\left(l_{1}+l_{2}\right)}, \ldots, X_{\left(l_{1}+l_{2}+\cdots+l_{k}\right)}$ is thus given, for $1 \leq k \leq n$, $1 \leq l_{1}+l_{2}+\cdots+l_{k} \leq n\left(l_{i} \geq 1\right)$ and $x_{1} \leq x_{2} \leq \cdots \leq x_{k}\left(x_{i} \in(0, t)\right)$ by

$$
\begin{aligned}
g_{l_{1}, l_{2}, \ldots, l_{k}} & \left(x_{1}, x_{2}, \ldots, x_{k}\right)= \\
& \frac{n !\left(\frac{1}{t}\right)^{k}\left(\frac{x_{1}}{t}\right)^{l_{1}-1}\left(\frac{x_{2}-x_{1}}{t}\right)^{l_{2}-1} \cdots\left(\frac{x_{k}-x_{k-1}}{t}\right)^{l_{k}-1}\left(1-\frac{x_{k}}{t}\right)^{n-\left(l_{1}+l_{2} \cdots+l_{k}\right)}}{\left(l_{1}-1\right) !\left(l_{2}-1\right) ! \cdots\left(l_{k}-1\right) !\left(n-\left(l_{1}+l_{2} \cdots+l_{k}\right)\right) !} .
\end{aligned}
$$

Furthermore, if we write $Y_{l_{1}}=X_{l_{1}}$ and for $i=2, \ldots, k$, we define $Y_{l_{i}}$ as $Y_{l_{i}}=$ $X_{\left(l_{1}+l_{2}+\cdots+l_{i}\right)}-X_{\left(l_{1}+l_{2}+\cdots+l_{i-1}\right)}$ then the joint density function of $Y_{l_{1}}, Y_{l_{2}}, \ldots, Y_{l_{k}}$ is given, for $1 \leq k \leq n, 1 \leq l_{1}+l_{2}+\cdots+l_{k} \leq n\left(l_{i} \geq 1\right)$ and $0<s_{1}+s_{2}+\cdots+s_{k}<t\left(s_{i} \in(0, t)\right)$ by

$$
h_{l_{1}, l_{2}, \ldots, l_{k}}\left(s_{1}, s_{2}, \ldots, s_{k}\right)=g_{l_{1}, l_{2}, \ldots, l_{k}}\left(s_{1}, s_{1}+s_{2}, \ldots, s_{1}+\cdots+s_{k}\right) \text {, }
$$

that is,

$$
\begin{aligned}
& h_{l_{1}, l_{2}, \ldots, l_{k}}\left(s_{1}, s_{2}, \ldots, s_{k}\right)= \\
& \quad \frac{n !\left(\frac{1}{t}\right)^{k}\left(\frac{s_{1}}{t}\right)^{l_{1}-1}\left(\frac{s_{2}}{t}\right)^{l_{2}-1} \cdots\left(\frac{s_{k}}{t}\right)^{l_{k}-1}\left(1-\frac{s_{1}+s_{2}+\cdots+s_{k}}{t}\right)^{n-\left(l_{1}+l_{2} \cdots+l_{k}\right)}}{\left(l_{1}-1\right) !\left(l_{2}-1\right) ! \cdots\left(l_{k}-1\right) !\left(n-\left(l_{1}+l_{2} \cdots+l_{k}\right)\right) !} .
\end{aligned}
$$

In particular, for $k=n$ we get the joint density function of the random variables $Y_{1}=X_{(1)}, Y_{2}=X_{(2)}-X_{(1)}, \ldots, Y_{n}=X_{(n)}-X_{(n-1)}$ denoted by $h\left(x_{1}, x_{2}, \ldots, x_{n}\right)$ by making $l_{1}=l_{2}=\cdots=l_{n}=1$ in the previous expression, that is

$$
h\left(x_{1}, x_{2}, \ldots, x_{n}\right)=\left\{\begin{array}{cc}
\frac{n !}{t^{n}} & \text { if } \sum_{i=1}^{n} x_{i} \leq t \\
0 & \text { otherwise. }
\end{array}\right.
$$

By writing $Y_{n+1}=t-X_{(n)}$, this also determines the (degenerate) joint density function of $Y_{1}, Y_{2}, \ldots, Y_{n}, Y_{n+1}$ in the region

$$
x_{i} \geq 0 \quad(i=1, \ldots, n, n+1) \quad \sum_{i=1}^{n+1} x_{i}=t .
$$

The joint distribution of $Y_{l_{1}}, Y_{l_{2}}, \ldots, Y_{l_{k}}$ is denoted by $H_{l_{1}, l_{2}, \ldots, l_{k}}\left(s_{1}, s_{2}, \ldots, s_{k}\right)$, that is

$$
H_{l_{1}, l_{2}, \ldots, l_{k}}\left(s_{1}, s_{2}, \ldots, s_{k}\right)=\mathbb{P}\left\{Y_{l_{1}} \leq s_{1}, Y_{l_{2}} \leq s_{2}, \ldots, Y_{l_{k}} \leq s_{k}\right\} .
$$

This distribution is given in the following lemma. 
Lemma 2.1 For $1 \leq k \leq n, 1 \leq l_{1}+l_{2}+\cdots+l_{k} \leq n\left(l_{i} \geq 1\right)$ and $0<s_{1}+s_{2}+\cdots+s_{k}<t$ $\left(s_{i} \in(0, t)\right)$, we have

$H_{l_{1}, l_{2}, \ldots, l_{k}}\left(s_{1}, s_{2}, \ldots, s_{k}\right)=$

$i_{1} \geq l_{1}, i_{2} \geq l_{2}, \ldots, i_{k} \geq l_{k}$

$$
\frac{n !\left(\frac{s_{1}}{t}\right)^{i_{1}}\left(\frac{s_{2}}{t}\right)^{i_{2}} \cdots\left(\frac{s_{k}}{t}\right)^{i_{k}}\left(1-\frac{s_{1}+s_{2}+\cdots+s_{k}}{t}\right)^{n-\left(i_{1}+i_{2} \cdots+i_{k}\right)}}{i_{1} ! i_{2} ! \cdots i_{k} !\left(n-\left(i_{1}+i_{2} \cdots+i_{k}\right)\right) !} .
$$

$i_{1}+i_{2}+\cdots+i_{k} \leq n$

Proof. It suffices to show that

$$
\frac{\partial^{k} H_{l_{1}, l_{2}, \ldots, l_{k}}\left(s_{1}, s_{2}, \ldots, s_{k}\right)}{\partial s_{1} \partial s_{2} \cdots \partial s_{k}}=h_{l_{1}, l_{2}, \ldots, l_{k}}\left(s_{1}, s_{2}, \ldots, s_{k}\right)
$$

To simplify writing, we define

$$
\theta_{n ; i_{1}, i_{2}, \ldots, i_{k}}^{t ; s_{1}, s_{2}, \ldots, s_{k}}=\frac{\left(\frac{s_{1}}{t}\right)^{i_{1}}\left(\frac{s_{2}}{t}\right)^{i_{2}} \cdots\left(\frac{s_{k}}{t}\right)^{i_{k}}\left(1-\frac{s_{1}+s_{2}+\cdots+s_{k}}{t}\right)^{n-\left(i_{1}+i_{2} \cdots+i_{k}\right)}}{i_{1} ! i_{2} ! \cdots i_{k} !\left(n-\left(i_{1}+i_{2} \cdots+i_{k}\right)\right) !}
$$

We thus have, from relation (3),

$$
\begin{aligned}
& H_{l_{1}, l_{2}, \ldots, l_{k}}\left(s_{1}, s_{2}, \ldots, s_{k}\right)=n ! \sum_{i_{1} \geq l_{1}, i_{2} \geq l_{2}, \ldots, i_{k} \geq l_{k},} \theta_{n ; i_{1}, i_{2}, \ldots, i_{k}}^{t ; s_{1}, s_{2}, \ldots, s_{k}} . \\
& i_{1} \geq l_{1}, i_{2} \geq l_{2}, \ldots, i_{k} \geq l_{k},
\end{aligned}
$$

We obtain

$$
\begin{aligned}
& \frac{\partial H_{l_{1}, l_{2}, \ldots, l_{k}}\left(s_{1}, s_{2}, \ldots, s_{k}\right)}{\partial s_{k}}=\frac{n !}{t} \sum_{\substack{i_{1} \geq l_{1}, i_{2} \geq l_{2}, \ldots, i_{k} \geq l_{k}, i_{1}+i_{2}+\cdots+i_{k} \leq n}} \theta_{n ; i_{1}, i_{2}, \ldots, i_{k-1}, i_{k}-1}^{t ; s_{1}, s_{2}, \ldots, s_{k}} \\
& -\frac{n !}{t} \sum_{i_{1} \geq l_{1}, i_{2} \geq l_{2}, \ldots, i_{k} \geq l_{k},} \theta_{n-1 ; i_{1}, i_{2}, \ldots, i_{k}}^{t ; s_{1}, s_{2}, \ldots, s_{k}} \\
& i_{1}+i_{2}+\cdots+i_{n} \leq n-1
\end{aligned}
$$

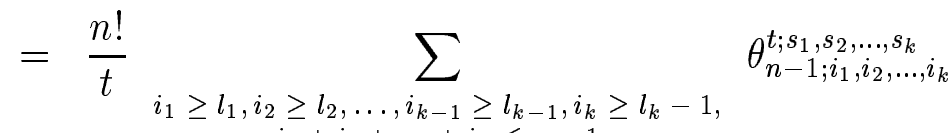

$$
\begin{aligned}
& i_{1}+i_{2}+\cdots+i_{k} \leq n-1
\end{aligned}
$$

INRIA 


$$
\begin{aligned}
& -\frac{n !}{t} \sum_{i_{1} \geq l_{1}, i_{2} \geq l_{2}, \ldots, i_{k} \geq l_{k}} \quad \theta_{n-1 ; i_{1}, i_{2}, \ldots, i_{k}}^{t ; s_{1}, s_{2}, \ldots, s_{k}} \\
& \begin{array}{c}
i_{1} \geq l_{1}, i_{2} \geq l_{2}, \ldots, i_{k} \geq l_{k} \\
i_{1}+i_{2}+\cdots+i_{k} \leq n-1
\end{array} \\
& =\frac{n !}{t} \sum_{\substack{i_{1} \geq l_{1}, i_{2} \geq l_{2}, \ldots, i_{k-1} \geq l_{k-1}, i_{1}+i_{2}+\cdots+i_{k-1} \leq n-1}} \theta_{n-1 ; i_{1}, i_{2}, \ldots, i_{k-1}, l_{k}-1}^{t ; s_{1}, s_{2}, \ldots, s_{k}}
\end{aligned}
$$

where the second equality is obtained by using the variable change $i_{k} \longrightarrow i_{k}+1$. By iterating successively the same argument with respect to variables $s_{k-1}, \ldots, s_{2}$, we obtain

$$
\frac{\partial^{k-1} H_{l_{1}, l_{2}, \ldots, l_{k}}\left(s_{1}, s_{2}, \ldots, s_{k}\right)}{\partial s_{k} \partial s_{k-1}, \cdots, \partial s_{2}}=\frac{n !}{t^{k-1}} \sum_{\substack{i_{1} \geq l_{1}, i_{1} \leq n-\left(l_{2}+\cdots+l_{k}\right)}} \theta_{n-k+1 ; i_{1}, l_{2}-1, \ldots, l_{k-1}-1, l_{k}-1}^{t ; s_{1}, s_{2}, \ldots, s_{k}},
$$

and finally,

$$
\begin{aligned}
& \frac{\partial^{k} H_{l_{1}, l_{2}, \ldots, l_{k}}\left(s_{1}, s_{2}, \ldots, s_{k}\right)}{\partial s_{k}, \cdots, \partial s_{2}, \partial s_{1}}=\frac{n !}{t^{k}} \sum_{\substack{i_{1} \geq l_{1}, i_{1} \leq n-\left(l_{2}+\cdots+l_{k}\right)}} \theta_{n-k+1 ; i_{1}-1, l_{2}-1, \ldots, l_{k}-1}^{t ; s_{1}, s_{2}, \ldots, s_{k}} \\
& i_{1} \leq n-\left(l_{2}+\cdots+l_{k}\right) \\
& -\frac{n !}{t^{k}} \sum_{\substack{i_{1} \geq l_{1}, i_{1} \leq n-\left(l_{2}+\cdots+l_{k}\right)-1}} \theta_{n-k ; i_{1}, l_{2}-1, \ldots, l_{k}-1}^{t ; s_{1}, s_{2}, \ldots, s_{k}} \\
& =\frac{n !}{t^{k}} \quad \sum_{i_{1} \geq l_{1}-1,} \theta_{n-k ; i_{1}, l_{2}-1, \ldots, l_{k}-1}^{t ; s_{1}, s_{2}, \ldots, s_{k}} \\
& i_{1} \leq n-\left(l_{2}+\cdots+l_{k}\right)-1 \\
& -\frac{n !}{t^{k}} \sum_{\substack{i_{1} \geq l_{1}, i_{1} \leq n-\left(l_{2}+\cdots+l_{k}\right)-1}}^{\substack{t ; s_{1}, s_{2}, \ldots, s_{k} \\
n-k ; i_{1}, l_{2}-1, \ldots, l_{k}-1}} \\
& =\frac{n !}{t^{k}} \theta_{n-k ; l_{1}-1, l_{2}-1, \ldots, l_{k}-1}^{t ; s_{1}, s_{2}, \ldots, s_{k}} \\
& =h_{l_{1}, l_{2}, \ldots, l_{k}}\left(s_{1}, s_{2}, \ldots, s_{k}\right)
\end{aligned}
$$

where the second equality is obtained by using the variable change $i_{1} \longrightarrow i_{1}+1$.

$\mathrm{RR} \mathrm{n}^{\circ} 3806$ 


\subsection{The Poisson Process}

Let $\left\{N_{t}, t \in \mathbb{R}\right\}$ be a Poisson process with rate $\lambda$ and let $T_{0}, T_{0}+T_{1}, \ldots, T_{0}+T_{1}+\cdots+T_{n-1}$ be the $n$ first instants of jumps of $\left\{N_{t}\right\}$ over [0,t). It is well-known, see [4] that the density of the conditional distribution of $T_{0}, T_{1}, \ldots, T_{n-1}$ given that $\left\{N_{t}=n\right\}$ is

$$
f\left(x_{0}, x_{1}, \ldots, x_{n-1}\right)=\left\{\begin{array}{cl}
\frac{n !}{t^{n}} & \text { if } \sum_{i=0}^{n-1} x_{i} \leq t \\
0 & \text { otherwise }
\end{array}\right.
$$

This is also, as seen in the previous subsection, the joint density of the order statistics from the uniform distribution over $(0, t)$.

If we write $T_{n}=t-\left(T_{0}+T_{1}+\cdots+T_{n-1}\right)$, this also determines the (degenerate) joint density function of $T_{0}, T_{1}, \ldots, T_{n-1}, T_{n}$ in the region

$$
x_{i} \geq 0 \quad(i=0, \ldots, n-1, n) \quad \sum_{i=0}^{n} x_{i}=t .
$$

The symmetric role played by the variables $x_{0}, x_{1}, \ldots, x_{n-1}, x_{n}$ shows that for any subset $\left\{i_{1}, i_{2}, \cdots, i_{n}\right\}$ of the set $\{0,1, \ldots, n-1, n\}$, we have $f\left(x_{i_{1}}, x_{i_{2}}, \ldots, x_{i_{n}}\right)=f\left(x_{0}, x_{1}, \ldots, x_{n-1}\right)$.

It follows from relation (1) that we have, for $1 \leq l \leq n,\left\{i_{1}, i_{2}, \cdots, i_{l}\right\} \subset\{0,1, \ldots, n-$ $1, n\}$ and $s \in(0, t)$,

$$
\begin{aligned}
\mathbb{P}\left\{T_{i_{1}}+\cdots+T_{i_{l}} \leq s \mid N_{t}=n\right\} & =\mathbb{P}\left\{T_{0}+\cdots+T_{l-1} \leq s \mid N_{t}=n\right\} \\
& =\mathbb{P}\left\{X_{(l)} \leq s\right\} \\
& =\sum_{k=l}^{n}\left(\begin{array}{l}
n \\
k
\end{array}\right)\left(\frac{s}{t}\right)^{k}\left(1-\frac{s}{t}\right)^{n-k}
\end{aligned}
$$

More generally, let $k$ be an integer such that $1 \leq k \leq n$ and $l_{1}, l_{2}, \ldots, l_{k}$ be $k$ integers such that $1 \leq l_{1}+l_{2}+\cdots+l_{k} \leq n\left(l_{i} \geq 1\right)$. For any subset $\left\{i_{1}, i_{2}, \ldots, i_{l_{1}+l_{2}+\cdots+l_{k}}\right\}$ of the $\{0,1, \ldots, n-1, n\}$, the vectors

$$
\left(\sum_{j=1}^{l_{1}} T_{i_{j}}, \sum_{j=l_{1}+1}^{l_{1}+l_{2}} T_{i_{j}}, \ldots, \sum_{j=l_{1}+l_{2}+\cdots+l_{k-1}+1}^{l_{1}+l_{2}+\cdots+l_{k}} T_{i_{j}}\right)
$$

and

$$
\left(\sum_{j=0}^{l_{1}-1} T_{j}, \sum_{j=l_{1}}^{l_{1}+l_{2}-1} T_{j}, \ldots, \sum_{j=l_{1}+l_{2}+\cdots+l_{k-1}}^{l_{1}+l_{2}+\cdots+l_{k}-1} T_{j}\right)
$$


have the same conditional distribution given that $N_{t}=n$, given by lemma 2.1 , that is, for $0<s_{1}+s_{2}+\cdots+s_{k}<t\left(s_{i} \in(0, t)\right)$,

$$
\begin{aligned}
\mathbb{P}\left\{\sum_{j=1}^{l_{1}} T_{j} \leq s_{1}, \sum_{j=l_{1}+1}^{l_{1}+l_{2}} T_{j} \leq s_{2}, \ldots, \sum_{j=l_{1}+l_{2}+\cdots+l_{k-1}+1}^{l_{1}+l_{2}+\cdots+l_{k}} T_{j} \leq s_{k} \mid N_{t}=n\right\}= \\
\sum_{\substack{i_{1} \geq l_{1}, i_{2} \geq l_{2}, \ldots, i_{k} \geq l_{k} \\
i_{1}+i_{2}+\cdots+i_{k} \leq n}} \frac{n !\left(\frac{s_{1}}{t}\right)^{i_{1}}\left(\frac{s_{2}}{t}\right)^{i_{2}} \cdots\left(\frac{s_{k}}{t}\right)^{i_{k}}\left(1-\frac{s_{1}+s_{2}+\cdots+s_{k}}{t}\right)^{n-\left(i_{1}+i_{2} \cdots+i_{k}\right)}}{i_{1} ! i_{2} ! \cdots i_{k} !\left(n-\left(i_{1}+i_{2} \cdots+i_{k}\right)\right) !} .
\end{aligned}
$$

\section{Distribution of Occupation Times}

Let $X=\left\{X_{u}, u \geq 0\right\}$ be a homogeneous Markov process with a finite state space $S$. The process $X$ is given by its infinitesimal generator $A$ and by its initial probability distribution $\alpha$. We denote by $Z=\left\{Z_{n}, n \geq 0\right\}$ the uniformized Markov chain [7] associated to the Markov process $X$, with the same initial distribution $\alpha$. Its transition probability matrix $P$ is related to the matrix $A$ by the relation $P=I+A / \lambda$, where $I$ is the identity matrix and $\lambda$ satisfies the relation $\lambda \geq \max \left\{-A_{i, i}, i \in S\right\}$. The rate $\lambda$ is the rate of the Poisson process $\left\{N_{u}, u \geq 0\right\}$, independent of $Z$, which counts the number of transitions of process $\left\{Z_{N_{u}}, u \geq 0\right\}$ over $[0, t)$. It is well-known that the processes $\left\{Z_{N_{u}}\right\}$ and $X$ are stochastically equivalent. We consider a partition $S=U \cup D(U \cap D=\emptyset)$ of the state space $S$ and we consider the occupation time of the subset $U$.

\subsection{The Discrete Time Case}

We consider the Markov chain $Z=\left\{Z_{n}, n \geq 0\right\}$ and we define the random variable $V_{n}$ by the total number of states of $U$ visited during the $n$ first transitions of $Z$, that is

$$
V_{n}=\sum_{k=0}^{n} 1_{\left\{Z_{k} \in U\right\}} .
$$

This random variable represents the occupation time of subset $U$ during the $n$ first transitions of the process $Z$. The following theorem gives the backward equation for the behavior of the couple $\left(V_{n}, Z_{n}\right)$. 
Theorem 3.1 For $n \geq 1$ and $1 \leq k \leq n$, we have

for $i \in U, \mathbb{P}\left\{V_{n} \leq k, Z_{n}=j \mid Z_{0}=i\right\}=\sum_{l \in E} P_{i, l} \mathbb{P}\left\{V_{n-1} \leq k-1, Z_{n-1}=j \mid Z_{0}=l\right\}$

for $i \in D, \mathbb{P}\left\{V_{n} \leq k, Z_{n}=j \mid Z_{0}=i\right\}=\sum_{l \in E} P_{i, l} \mathbb{P}\left\{V_{n-1} \leq k, Z_{n-1}=j \mid Z_{0}=l\right\}$

Proof. We have

$$
\begin{aligned}
\mathbb{P}\left\{V_{n} \leq k, Z_{n}\right. & \left.=j \mid Z_{0}=i\right\} \\
& =\sum_{l \in E} P_{i, l} \mathbb{P}\left\{V_{n} \leq k, Z_{n}=j \mid Z_{1}=l, Z_{0}=i\right\} \\
& =\sum_{l \in E} P_{i, l} \mathbb{P}\left\{\sum_{h=1}^{n} 1_{\left\{Z_{h} \in U\right\}} \leq k-1_{\{i \in U\}}, Z_{n}=j \mid Z_{1}=l, Z_{0}=i\right\} \\
& =\sum_{l \in E} P_{i, l} \mathbb{P}\left\{\sum_{h=1}^{n} 1_{\left\{Z_{h} \in U\right\}} \leq k-1_{\{i \in U\}}, Z_{n}=j \mid Z_{1}=l\right\} \\
& =\sum_{l \in E} P_{i, l} \mathbb{P}\left\{\sum_{h=0}^{n-1} 1_{\left\{Z_{h} \in U\right\}} \leq k-1_{\{i \in U\}}, Z_{n-1}=j \mid Z_{0}=l\right\} \\
& =\sum_{l \in E} P_{i, l} \mathbb{P}\left\{V_{n-1} \leq k-1_{\{i \in U\}}, Z_{n-1}=j \mid Z_{0}=l\right\}
\end{aligned}
$$

The third equality follows from the Markov property of $Z$ and the fourth follows from the homogeneity of $Z$.

The following theorem gives the forward equation for the behavior of the couple $\left(V_{n}, Z_{n}\right)$.

Theorem 3.2 For $n \geq 1$ and $1 \leq k \leq n$, we have

$$
\begin{aligned}
& \text { for } j \in U, \mathbb{P}\left\{V_{n} \leq k, Z_{n}=j \mid Z_{0}=i\right\}=\sum_{l \in E} \mathbb{P}\left\{V_{n-1} \leq k-1, Z_{n-1}=l \mid Z_{0}=i\right\} P_{l, j} \\
& \text { for } j \in D, \mathbb{P}\left\{V_{n} \leq k, Z_{n}=j \mid Z_{0}=i\right\}=\sum_{l \in E} \mathbb{P}\left\{V_{n-1} \leq k, Z_{n-1}=l \mid Z_{0}=i\right\} P_{l, j}
\end{aligned}
$$

Proof. We have 


$$
\begin{aligned}
\mathbb{P}\left\{V_{n}\right. & \left.\leq k, Z_{n}=j, Z_{0}=i\right\} \\
& =\mathbb{P}\left\{V_{n-1} \leq k-1_{\{j \in U\}}, Z_{n}=j, Z_{0}=i\right\} \\
& =\sum_{l \in E} \mathbb{P}\left\{V_{n-1} \leq k-1_{\{j \in U\}}, Z_{n}=j, Z_{n-1}=l, Z_{0}=i\right\} \\
& =\sum_{l \in E} \mathbb{P}\left\{V_{n-1} \leq k-1_{\{j \in U\}}, Z_{0}=i \mid Z_{n}=j, Z_{n-1}=l\right\} \mathbb{P}\left\{Z_{n}=j, Z_{n-1}=l\right\} \\
& =\sum_{l \in E} \mathbb{P}\left\{V_{n-1} \leq k-1_{\{j \in U\}}, Z_{0}=i \mid Z_{n-1}=l\right\} \mathbb{P}\left\{Z_{n}=j, Z_{n-1}=l\right\} \\
& =\sum_{l \in E} \mathbb{P}\left\{V_{n-1} \leq k-1_{\{j \in U\}}, Z_{n-1}=l, Z_{0}=i\right\} \mathbb{P}\left\{Z_{n}=j \mid Z_{n-1}=l\right\} \\
& =\sum_{l \in E} \mathbb{P}\left\{V_{n-1} \leq k-1_{\{j \in U\}}, Z_{n-1}=l, Z_{0}=i\right\} P_{l, j}
\end{aligned}
$$

The fourth equality follows from the Markov property of $Z$ and the last one follows from the homogeneity of $Z$. We thus obtain the desired relation by conditioning with respect to $Z_{0}$.

If we denote, for $n \geq 0$ and $k \geq 0$, by $F(n, k)$ the matrix whose entry $(i, j)$ is defined by

$$
F_{i, j}(n, k)=\mathbb{P}\left\{V_{n} \leq k, Z_{n}=j \mid Z_{0}=i\right\},
$$

the results of theorems 3.1 and 3.2 can be easily expressed in matrix notation. We first decompose the matrices $P$ and $F(n, k)$ with respect to the partition $\{U, D\}$ of the state space $S$ as

$$
P=\left(\begin{array}{cc}
P_{U} & P_{U D} \\
P_{D U} & P_{D}
\end{array}\right) \quad \text { and } \quad F(n, k)=\left(\begin{array}{cc}
F_{U}(n, k) & F_{U D}(n, k) \\
F_{D U}(n, k) & F_{D}(n, k)
\end{array}\right) .
$$

The result of theorem 3.1 can then be written as

$$
\begin{aligned}
\left(\begin{array}{ll}
F_{U}(n, k) & F_{U D}(n, k)
\end{array}\right) & =\left(\begin{array}{cc}
P_{U} & P_{U D}
\end{array}\right) F(n-1, k-1) \\
\left(\begin{array}{lll}
F_{D U}(n, k) & F_{D}(n, k)
\end{array}\right) & =\left(\begin{array}{cc}
P_{D U} & P_{D}
\end{array}\right) F(n-1, k)
\end{aligned}
$$

or also as

$$
F(n, k)=\left(\begin{array}{cc}
P_{U} & P_{U D} \\
0 & 0
\end{array}\right) F(n-1, k-1)+\left(\begin{array}{cc}
0 & 0 \\
P_{D U} & P_{D}
\end{array}\right) F(n-1, k) .
$$

$\mathrm{RR} \mathrm{n}^{\circ} 3806$ 
In the same way, the result of theorem 3.2 can be written as

$$
\begin{aligned}
& \left(\begin{array}{c}
F_{U}(n, k) \\
F_{D U}(n, k)
\end{array}\right)=F(n-1, k-1)\left(\begin{array}{c}
P_{U} \\
P_{D U}
\end{array}\right) \\
& \left(\begin{array}{c}
F_{U D}(n, k) \\
F_{D}(n, k)
\end{array}\right)=F(n-1, k)\left(\begin{array}{c}
P_{U D} \\
P_{D}
\end{array}\right)
\end{aligned}
$$

or also as

$$
F(n, k)=F(n-1, k-1)\left(\begin{array}{cc}
P_{U} & 0 \\
P_{D U} & 0
\end{array}\right)+F(n-1, k)\left(\begin{array}{cc}
0 & P_{U D} \\
0 & P_{D}
\end{array}\right) .
$$

The initial conditions are given, for $n \geq 0$, by

$$
F(n, 0)=\left(\begin{array}{cc}
0 & 0 \\
0 & \left(P_{D}\right)^{n}
\end{array}\right)
$$

Note also that we have for every $k \geq n+1$

$$
F(n, k)=P^{n}
$$

\subsection{The Continuous Time Case}

We consider now the Markov process $X=\left\{X_{t}, t \geq 0\right\}$ and the occupation time $W_{t}$ of the subset $U$ over $[0, t)$, that is

$$
W_{t}=\int_{0}^{t} 1_{\left\{X_{u} \in U\right\}}
$$

This random variable represents the time spent by the process $X$ in the subset $U$ during the interval $[0, t)$. The joint distribution of the couple $\left(W_{t}, X_{t}\right)$ is given by the following theorem. The notation $\mathbb{P}_{i}$ denotes the conditional probability given that $X_{0}=i$, that is $\mathbb{P}_{i}\{\cdot\}=\mathbb{P}\left\{\cdot \mid X_{0}=i\right\}$.

Theorem 3.3 For every $i, j \in S$, for $t>0$ and $s \in[0, t)$, we have

$$
\begin{aligned}
\mathbb{P}\left\{W_{t} \leq s, X_{t}=j \mid X_{0}=i\right\} & \\
& =\sum_{n=0}^{\infty} e^{-\lambda t} \frac{(\lambda t)^{n}}{n !} \sum_{k=0}^{n}\left(\begin{array}{l}
n \\
k
\end{array}\right)\left(\frac{s}{t}\right)^{k}\left(1-\frac{s}{t}\right)^{n-k} \mathbb{P}\left\{V_{n} \leq k, Z_{n}=j \mid Z_{0}=i\right\}
\end{aligned}
$$


Proof. We have, for $s<t$,

$$
\begin{aligned}
& \mathbb{P}\left\{W_{t} \leq s, X_{t}=j \mid X_{0}=i\right\} \\
& =\sum_{n=0}^{\infty} \mathbb{P}_{i}\left\{W_{t} \leq s, N_{t}=n, X_{t}=j\right\} \\
& =\sum_{n=0}^{\infty} \mathbb{P}_{i}\left\{W_{t} \leq s, N_{t}=n, Z_{n}=j\right\} \quad \text { since }\left\{X_{t}\right\} \text { and }\left\{Z_{N_{t}}\right\} \text { are equivalent } \\
& =\sum_{n=0}^{\infty} \mathbb{P}_{i}\left\{N_{t}=n\right\} \mathbb{P}_{i}\left\{W_{t} \leq s, Z_{n}=j \mid N_{t}=n\right\} \\
& =\sum_{n=0}^{\infty} \mathbb{P}\left\{N_{t}=n\right\} \mathbb{P}_{i}\left\{W_{t} \leq s, Z_{n}=j \mid N_{t}=n\right\} \\
& =\sum_{n=0}^{\infty} \mathbb{P}\left\{N_{t}=n\right\} \sum_{l=0}^{n+1} \mathbb{P}_{i}\left\{W_{t} \leq s, V_{n}=l, Z_{n}=j \mid N_{t}=n\right\} \\
& =\sum_{n=0}^{\infty} \mathbb{P}\left\{N_{t}=n\right\} \sum_{l=0}^{n+1} \mathbb{P}_{i}\left\{V_{n}=l, Z_{n}=j \mid N_{t}=n\right\} \mathbb{P}_{i}\left\{W_{t} \leq s \mid V_{n}=l, Z_{n}=j, N_{t}=n\right\} \\
& =\sum_{n=0}^{\infty} \mathbb{P}\left\{N_{t}=n\right\} \sum_{l=0}^{n+1} \mathbb{P}_{i}\left\{V_{n}=l, Z_{n}=j\right\} \mathbb{P}_{i}\left\{W_{t} \leq s \mid V_{n}=l, Z_{n}=j, N_{t}=n\right\} \\
& =\sum_{n=0}^{\infty} \mathbb{P}\left\{N_{t}=n\right\} \sum_{l=0}^{n} \mathbb{P}_{i}\left\{V_{n}=l, Z_{n}=j\right\} \mathbb{P}_{i}\left\{W_{t} \leq s \mid V_{n}=l, Z_{n}=j, N_{t}=n\right\}
\end{aligned}
$$

Note that the 4 th and the 7 th equalities follow from the independence of the processes $\left\{Z_{n}\right\}$ and $\left\{N_{t}\right\}$ and the fact that $X_{0}=Z_{0}$. The last equality follows from the fact if $l=n+1$, we trivially obtain that $V_{n}=n+1$ and $N_{t}=n$ imply that $W_{t}=t$ and so we get $\mathbb{P}\left\{W_{t} \leq s \mid V_{n}=n+1, Z_{n}=j, N_{t}=n\right\}=0$ since we have supposed that $s<t$.

Let us consider now the expression $\mathbb{P}_{i}\left\{W_{t} \leq s \mid V_{n}=l, Z_{n}=j, N_{t}=n\right\}$.

For fixed $i, j \in S$ and $0 \leq l \leq n$, we define the set

$$
G_{l, n}^{i, j}=\left\{\widehat{z}=\left(i, z_{1}, \ldots, z_{n-1}, j\right) \in S^{n+1} \mid l \text { entries of } \widehat{z} \text { are in } U \text { and } n+1-l \text { are in } D\right\}
$$

and we denote by $\widehat{Z}$ the random vector $\left(Z_{0}, \ldots, Z_{n}\right)$. We then have 


$$
\begin{aligned}
\mathbb{P}_{i} & \left\{W_{t} \leq s \mid V_{n}=l, Z_{n}=j, N_{t}=n\right\} \\
& =\sum_{\widehat{z} \in G_{l, n}^{i, j}} \mathbb{P}_{i}\left\{W_{t} \leq s \mid \widehat{Z}=\widehat{z}, V_{n}=l, Z_{n}=j, N_{t}=n\right\} \mathbb{P}_{i}\left\{\widehat{Z}=\widehat{z} \mid V_{n}=l, Z_{n}=j, N_{t}=n\right\} \\
& =\sum_{\widehat{z} \in G_{l, n}^{i, j}} \mathbb{P}\left\{W_{t} \leq s \mid \widehat{Z}=\widehat{z}, V_{n}=l, N_{t}=n\right\} \mathbb{P}\left\{\widehat{Z}=\widehat{z} \mid V_{n}=l, Z_{n}=j, Z_{0}=i, N_{t}=n\right\} \\
& =\sum_{\widehat{z} \in G_{l, n}^{i, j}} \mathbb{P}\left\{W_{t} \leq s \mid \widehat{Z}=\widehat{z}, V_{n}=l, N_{t}=n\right\} \mathbb{P}\left\{\widehat{Z}=\widehat{z} \mid V_{n}=l, Z_{n}=j, Z_{0}=i\right\}
\end{aligned}
$$

where the last equality follows from the independence of the processes $\left\{Z_{n}\right\}$ and $\left\{N_{t}\right\}$. We denote by $T_{0}, T_{0}+T_{1}, \ldots, T_{0}+T_{1}+\cdots+T_{n-1}$ the $n$ first instants of jumps of the Poisson process $\left\{N_{t}\right\}$ over $[0, t)$ and we define $T_{n}=t-\left(T_{0}+T_{1}+\cdots+T_{n-1}\right)$. We then have

$$
\begin{aligned}
\mathbb{P}\left\{W_{t} \leq s \mid \widehat{Z}=\widehat{z}, V_{n}=l, N_{t}=n\right\} & =\mathbb{P}\left\{T_{i_{1}}+\cdots+T_{i_{l}} \leq s \mid \widehat{Z}=\widehat{z}, V_{n}=l, N_{t}=n\right\} \\
& =\mathbb{P}\left\{T_{i_{1}}+\cdots+T_{i_{l}} \leq s \mid N_{t}=n\right\}
\end{aligned}
$$

where the distinct indices $i_{1}, \ldots, i_{l} \in\{0,1, \ldots, n\}$ correspond to the $l$ entries of $\widehat{z}$ that are in $U$ and the last equality is due to the independence of the processes $\left\{Z_{n}\right\}$ and $\left\{N_{t}\right\}$. Note that for $l=0$, we obtain the correct result, which is equal to 1 by using the convention $\sum_{a}^{b}(\ldots)=0$ if $a>b$.

From relation (2) we get, for $l=0, \ldots, n$,

$$
\begin{aligned}
\mathbb{P}\left\{T_{i_{1}}+\cdots+T_{i_{l}} \leq s \mid N_{t}=n\right\} & =\mathbb{P}\left\{T_{0}+\cdots+T_{l-1} \leq s \mid N_{t}=n\right\} \\
& =\sum_{k=l}^{n}\left(\begin{array}{l}
n \\
k
\end{array}\right)\left(\frac{s}{t}\right)^{k}\left(1-\frac{s}{t}\right)^{n-k}
\end{aligned}
$$

Again, the use of the convention $\sum_{a}^{b}(\ldots)=0$ if $a>b$ allows us to consider both cases $l=0$ and $l=n+1$ as normal ones. Finally, we obtain

$$
\begin{aligned}
\mathbb{P}_{i}\left\{W_{t}\right. & \left.\leq s \mid V_{n}=l, Z_{n}=j, N_{t}=n\right\} \\
& =\sum_{\widehat{z} \in G_{l, n}^{i, j}} \sum_{k=l}^{n}\left(\begin{array}{l}
n \\
k
\end{array}\right)\left(\frac{s}{t}\right)^{k}\left(1-\frac{s}{t}\right)^{n-k} \mathbb{P}\left\{\widehat{Z}=\widehat{z} \mid V_{n}=l, Z_{n}=j, Z_{0}=i\right\} \\
& =\sum_{k=l}^{n}\left(\begin{array}{l}
n \\
k
\end{array}\right)\left(\frac{s}{t}\right)^{k}\left(1-\frac{s}{t}\right)^{n-k}
\end{aligned}
$$


That is, since $\mathbb{P}\left\{N_{t}=n\right\}=e^{-\lambda t} \frac{(\lambda t)^{n}}{n !}$,

$$
\begin{aligned}
\mathbb{P}\left\{W_{t}\right. & \left.\leq s, X_{t}=j \mid X_{0}=i\right\} \\
& =\sum_{n=0}^{\infty} e^{-\lambda t} \frac{(\lambda t)^{n}}{n !} \sum_{l=0}^{n} \sum_{k=l}^{n}\left(\begin{array}{l}
n \\
k
\end{array}\right)\left(\frac{s}{t}\right)^{k}\left(1-\frac{s}{t}\right)^{n-k} \mathbb{P}\left\{V_{n}=l, Z_{n}=j \mid Z_{0}=i\right\} \\
& =\sum_{n=0}^{\infty} e^{-\lambda t} \frac{(\lambda t)^{n}}{n !} \sum_{k=0}^{n}\left(\begin{array}{l}
n \\
k
\end{array}\right)\left(\frac{s}{t}\right)^{k}\left(1-\frac{s}{t}\right)^{n-k} \sum_{l=0}^{k} \mathbb{P}\left\{V_{n}=l, Z_{n}=j \mid Z_{0}=i\right\} \\
& =\sum_{n=0}^{\infty} e^{-\lambda t} \frac{(\lambda t)^{n}}{n !} \sum_{k=0}^{n}\left(\begin{array}{l}
n \\
k
\end{array}\right)\left(\frac{s}{t}\right)^{k}\left(1-\frac{s}{t}\right)^{n-k} \mathbb{P}\left\{V_{n} \leq k, Z_{n}=j \mid Z_{0}=i\right\}
\end{aligned}
$$

and the proof is completed.

Corollary 3.4 For $t>0$ and $s \in[0, t)$, we have

$$
\mathbb{P}\left\{W_{t} \leq s\right\}=\sum_{n=0}^{\infty} e^{-\lambda t} \frac{(\lambda t)^{n}}{n !} \sum_{k=0}^{n}\left(\begin{array}{l}
n \\
k
\end{array}\right)\left(\frac{s}{t}\right)^{k}\left(1-\frac{s}{t}\right)^{n-k} \mathbb{P}\left\{V_{n} \leq k\right\}
$$

Proof. We have, for $t>0$ and $s \in[0, t)$,

$$
\mathbb{P}\left\{W_{t} \leq s\right\}=\sum_{i \in S} \mathbb{P}\left\{X_{0}=i\right\} \sum_{j \in S} \mathbb{P}\left\{W_{t} \leq s, X_{t}=j \mid X_{0}=i\right\}
$$

and for $n \geq 0$ and $0 \leq k \leq n$,

$$
\mathbb{P}\left\{V_{n} \leq k\right\}=\sum_{i \in S} \mathbb{P}\left\{Z_{0}=i\right\} \sum_{j \in S} \mathbb{P}\left\{V_{n} \leq k, Z_{n}=j \mid Z_{0}=i\right\} .
$$

The result easily follows from theorem 3.3 and because $X_{0}=Z_{0}$.

From relation (4), for $t>0$, the distribution $\mathbb{P}\left\{W_{t} \leq s, X_{t}=j \mid X_{0}=i\right\}$ is differentiable with respect to $s$ for $s \in(0, t)$ and its derivative is given by the following corollary.

Corollary 3.5 For $t>0$ and $s \in(0, t)$, 


$$
\begin{aligned}
& \frac{d \mathbb{P}\left\{W_{t} \leq s, X_{t}=j \mid X_{0}=i\right\}}{d s} \\
& =\lambda \sum_{n=0}^{\infty} e^{-\lambda t} \frac{(\lambda t)^{n}}{n !} \sum_{k=0}^{n}\left(\begin{array}{l}
n \\
k
\end{array}\right)\left(\frac{s}{t}\right)^{k}\left(1-\frac{s}{t}\right)^{n-k} \mathbb{P}\left\{V_{n+1}=k+1, Z_{n+1}=j \mid Z_{0}=i\right\}
\end{aligned}
$$

Proof. In order to simplify writing let $G_{i, j}(t, s)=\mathbb{P}\left\{W_{t} \leq s, X_{t}=j \mid X_{0}=i\right\}$ and $F_{i, j}(n, k)=\mathbb{P}\left\{V_{n} \leq k, Z_{n}=j \mid Z_{0}=i\right\}$ and $f_{i, j}(n, k)=\mathbb{P}\left\{V_{n}=k, Z_{n}=j \mid Z_{0}=i\right\}$. From relation (4), for $t>0$ and $s \in(0, t)$ we have

$$
\begin{aligned}
\frac{d G_{i, j}(t, s)}{d s}= & \frac{1}{t} \sum_{n=1}^{\infty} e^{-\lambda t} \frac{(\lambda t)^{n}}{n !} \sum_{k=1}^{n} \frac{n !}{(k-1) !(n-k) !}\left(\frac{s}{t}\right)^{k-1}\left(1-\frac{s}{t}\right)^{n-k} F_{i, j}(n, k) \\
& -\frac{1}{t} \sum_{n=1}^{\infty} e^{-\lambda t} \frac{(\lambda t)^{n}}{n !} \sum_{k=0}^{n-1} \frac{n !}{k !(n-k-1) !}\left(\frac{s}{t}\right)^{k}\left(1-\frac{s}{t}\right)^{n-k-1} F_{i, j}(n, k) \\
= & \frac{1}{t} \sum_{n=1}^{\infty} e^{-\lambda t} \frac{(\lambda t)^{n}}{n !} \sum_{k=0}^{n-1} \frac{n !}{k !(n-k-1) !}\left(\frac{s}{t}\right)^{k}\left(1-\frac{s}{t}\right)^{n-k-1} F_{i, j}(n, k+1) \\
& -\frac{1}{t} \sum_{n=1}^{\infty} e^{-\lambda t} \frac{(\lambda t)^{n}}{n !} \sum_{k=0}^{n-1} \frac{n !}{k !(n-k-1) !}\left(\frac{s}{t}\right)^{k}\left(1-\frac{s}{t}\right)^{n-k-1} F_{i, j}(n, k) \\
= & \frac{1}{t} \sum_{n=1}^{\infty} e^{-\lambda t} \frac{(\lambda t)^{n}}{n !} \sum_{k=0}^{n-1} \frac{n !}{k !(n-k-1) !}\left(\frac{s}{t}\right)^{k}\left(1-\frac{s}{t}\right)^{n-k-1} f_{i, j}(n, k+1) \\
= & \lambda \sum_{n=0}^{\infty} e^{-\lambda t} \frac{(\lambda t)^{n}}{n !} \sum_{k=0}^{n}\left(\begin{array}{l}
n \\
k
\end{array}\right)\left(\frac{s}{t}\right)^{k}\left(1-\frac{s}{t}\right)^{n-k} f_{i, j}(n+1, k+1),
\end{aligned}
$$

which completes the proof.

\section{Joint Distribution of Occupation Times}

We consider now a partition of the state space $S$ in $m+1$ non empty subsets $B_{0}, B_{1}, \ldots$, $B_{m}$. We thus have $B_{i} \cap B_{j}=\emptyset$ for $i \neq j$ and $S=B_{0} \cup B_{1} \cup \cdots \cup B_{m}$. 


\subsection{The Discrete Time Case}

We consider the random variables $V_{n}^{i}$ defined by

$$
V_{n}^{i}=\sum_{k=0}^{n} 1_{\left\{Z_{k} \in B_{i}\right\}} .
$$

The following theorem gives the backward equation for the joint distribution of the $V_{n}^{i}$ and $Z_{n}$.

Theorem 4.1 For $r=1, \ldots, m$ and $n \geq 1$ and $1 \leq k_{1}, \ldots, k_{m} \leq n$ we have

$$
\begin{aligned}
\text { for } i \in B_{r}, \mathbb{P}\left\{V_{n}^{1} \leq k_{1}, \ldots, V_{n}^{r} \leq k_{r}, \ldots, V_{n}^{m} \leq k_{m}, Z_{n}=j \mid Z_{0}=i\right\}= \\
\qquad \sum_{l \in E} P_{i, l} \mathbb{P}\left\{V_{n-1}^{1} \leq k_{1}, \ldots, V_{n-1}^{r} \leq k_{r}-1, \ldots, V_{n-1}^{m} \leq k_{m}, Z_{n-1}=j \mid Z_{0}=l\right\}
\end{aligned}
$$

for $i \in B_{0}, \mathbb{P}\left\{V_{n}^{1} \leq k_{1}, \ldots, V_{n}^{m} \leq k_{m}, Z_{n}=j \mid Z_{0}=i\right\}=$

$$
\sum_{l \in E} P_{i, l} \mathbb{P}\left\{V_{n-1}^{1} \leq k_{1}, \ldots, V_{n-1}^{m} \leq k_{m}, Z_{n-1}=j \mid Z_{0}=l\right\}
$$

Proof. We denote by $\widehat{V_{n}}$ and $\widehat{k}$ the vectors $\left(V_{n}^{1}, \ldots, V_{n}^{m}\right)$ and $\left(k_{1}, \ldots, k_{m}\right)$ respectively and by $e_{i}, i=1, \ldots, m$, the row vector of dimension $m$ whose $i$ th entry is 1 and the others 0 . The proof can be done using the same steps used in the proof of theorem 3.1.

We have

$$
\begin{aligned}
\mathbb{P}\left\{\widehat{V_{n}} \leq \widehat{k}, Z_{n}=j \mid Z_{0}=i\right\} & =\sum_{l \in E} P_{i, l} \mathbb{P}\left\{\widehat{V_{n}} \leq \widehat{k}, Z_{n}=j \mid Z_{1}=l, Z_{0}=i\right\} \\
& =\sum_{l \in E} P_{i, l} \mathbb{P}\left\{\widehat{V_{n-1}} \leq \widehat{k}-e_{r} 1_{\left\{i \in B_{r}\right\}}, Z_{n-1}=j \mid Z_{0}=l\right\}
\end{aligned}
$$

The following theorem gives the forward equation for the joint distribution of the $V_{n}^{i}$ and $Z_{n}$.

Theorem 4.2 For $r=1, \ldots, m$ and $n \geq 1$ and $1 \leq k_{1}, \ldots, k_{m} \leq n$ we have 
for $j \in B_{r}, \mathbb{P}\left\{V_{n}^{1} \leq k_{1}, \ldots, V_{n}^{r} \leq k_{r}, \ldots, V_{n}^{m} \leq k_{m}, Z_{n}=j \mid Z_{0}=i\right\}=$

$$
\sum_{l \in E} \mathbb{P}\left\{V_{n-1}^{1} \leq k_{1}, \ldots, V_{n-1}^{r} \leq k_{r}-1, \ldots, V_{n-1}^{m} \leq k_{m}, Z_{n-1}=l \mid Z_{0}=i\right\} P_{l, j}
$$

for $j \in B_{0}, \mathbb{P}\left\{V_{n}^{1} \leq k_{1}, \ldots, V_{n}^{m} \leq k_{m}, Z_{n}=j \mid Z_{0}=i\right\}=$

$$
\sum_{l \in E} \mathbb{P}\left\{V_{n-1}^{1} \leq k_{1}, \ldots, V_{n-1}^{m} \leq k_{m}, Z_{n-1}=l \mid Z_{0}=i\right\} P_{l, j}
$$

Proof. With the notation of the proof of theorem 4.1, we follow the same steps used in the proof of theorem 3.2.

We have

$$
\begin{aligned}
\mathbb{P} & \left\{\widehat{V_{n}} \leq \widehat{k}, Z_{n}=j, Z_{0}=i\right\} \\
& =\mathbb{P}\left\{\widehat{V_{n-1}} \leq \widehat{k}-e_{r} 1_{\left\{j \in B_{r}\right\}}, Z_{n}=j, Z_{0}=i\right\} \\
& =\sum_{l \in E} \mathbb{P}\left\{\widehat{V_{n-1}} \leq \widehat{k}-e_{r} 1_{\left\{j \in B_{r}\right\}}, Z_{n}=j, Z_{n-1}=l, Z_{0}=i\right\} \\
& =\sum_{l \in E} \mathbb{P}\left\{\widehat{V_{n-1}} \leq \widehat{k}-e_{r} 1_{\left\{j \in B_{r}\right\}}, Z_{0}=i \mid Z_{n}=j, Z_{n-1}=l\right\} \mathbb{P}\left\{Z_{n}=j, Z_{n-1}=l\right\} \\
& =\sum_{l \in E} \mathbb{P}\left\{\widehat{V_{n-1}} \leq \widehat{k}-e_{r} 1_{\left\{j \in B_{r}\right\}}, Z_{0}=i \mid Z_{n-1}=l\right\} \mathbb{P}\left\{Z_{n}=j, Z_{n-1}=l\right\} \\
& =\sum_{l \in E} \mathbb{P}\left\{\widehat{V_{n-1}} \leq \widehat{k}-e_{r} 1_{\left\{j \in B_{r}\right\}}, Z_{n-1}=l, Z_{0}=i\right\} \mathbb{P}\left\{Z_{n}=j \mid Z_{n-1}=l\right\} \\
& =\sum_{l \in E} \mathbb{P}\left\{\widehat{V_{n-1}} \leq \widehat{k}-e_{r} 1_{\left\{j \in B_{r}\right\}}, Z_{n-1}=l, Z_{0}=i\right\} P_{l, j}
\end{aligned}
$$

The desired relation is then obtained by conditioning with respect to $Z_{0}$.

If we denote, for $n \geq 0$ and $k \geq 0$, by $F\left(n, k_{1}, \ldots, k_{m}\right)$ the matrix whose entry $(i, j)$ is defined by

$$
F_{i, j}\left(n, k_{1}, \ldots, k_{m}\right)=\mathbb{P}\left\{V_{n}^{1} \leq k_{1}, \ldots, V_{n}^{m} \leq k_{m}, Z_{n}=j \mid Z_{0}=i\right\},
$$

the results of theorems 4.1 and 4.2 can be easily expressed in matrix notation. We first decompose the matrices $P$ and $F\left(n, k_{1}, \ldots, k_{m}\right)$ with respect to the partition $\left\{B_{0}, B_{1}, \ldots, B_{m}\right\}$ of the state space $S$ as

$$
P=\left(P_{B_{r} B_{h}}\right)_{0 \leq r, h \leq m} \quad \text { and } \quad F\left(n, k_{1}, \ldots, k_{m}\right)=\left(F_{B_{r} B_{h}}\left(n, k_{1}, \ldots, k_{m}\right)\right)_{0 \leq r, h \leq m} .
$$


The result of theorem 4.1 can then be written as

$$
F_{B_{r} B_{h}}\left(n, k_{1}, \ldots, k_{m}\right)=\sum_{l=0}^{m} P_{B_{r} B_{l}} F_{B_{l} B_{h}}\left(n-1, k_{1}, \ldots, k_{r}-1_{\{r \neq 0\}}, \ldots, k_{m}\right) .
$$

In the same way, the result of theorem 3.2 can be written as

$$
F_{B_{r} B_{h}}\left(n, k_{1}, \ldots, k_{m}\right)=\sum_{l=0}^{m} F_{B_{r} B_{l}}\left(n-1, k_{1}, \ldots, k_{h}-1_{\{h \neq 0\}}, \ldots, k_{m}\right) P_{B_{l} B_{h}} .
$$

The initial conditions are given, for $n \geq 0$, by

$$
F(n, 0, \ldots, 0)=\left(\begin{array}{cc}
0 & 0 \\
0 & \left(P_{B_{0} B_{0}}\right)^{n}
\end{array}\right),
$$

Note that in the case where $k_{1}+\cdots+k_{m} \geq n+1$, with $k_{i} \leq n$ for $i=1, \ldots, m$, the $m$ dimensional joint distribution of $V_{n}^{1}, \ldots, V_{n}^{m}$ can be expressed as a combination of $h$ dimensional joint distribution of the $V_{n}^{i}$ for $h=1, \ldots, m-1$. This observation is based on the following general result.

For any random variables $U_{1}, \ldots, U_{m}$ and any event $A$, we have

$$
\begin{aligned}
\mathbb{P}\left\{U_{1} \leq x_{1}, \ldots, U_{m} \leq x_{m}, A\right\}= & \sum_{E \subset\{1, \ldots, m\}}(-1)^{m-|E|+1} \mathbb{P}\left\{U_{i} \leq x_{i} ; i \in E, A\right\} \\
& +(-1)^{m} \mathbb{P}\left\{U_{1}>x_{1}, \ldots, U_{m}>x_{m}, A\right\}
\end{aligned}
$$

where the inclusion is strict, that is $E \neq\{1, \ldots, m\}$, and where we define for convenience $\mathbb{P}\left\{U_{i} \leq x_{i} ; i \in \emptyset, A\right\}=\mathbb{P}\{A\}$.

For what concerns our random variables $V_{n}^{1}, \ldots, V_{n}^{m}$, we have trivially

$$
\mathbb{P}\left\{V_{n}^{1}>k_{1}, \ldots, V_{n}^{m}>k_{m}, X_{n}=j \mid Z_{0}=i\right\}=0 \quad \text { if } \quad k_{1}+\cdots+k_{m} \geq n+1
$$

so we get in this case the desired result, that is

$$
\mathbb{P}_{i}\left\{V_{n}^{1} \leq k_{1}, \ldots, V_{n}^{m} \leq k_{m}, Z_{n}=j\right\}=\sum_{E \subset\{1, \ldots, m\}}(-1)^{m-|E|+1} \mathbb{P}_{i}\left\{V_{n}^{i} \leq k_{i} ; i \in E, Z_{n}=j\right\}
$$




\subsection{The Continuous Time Case}

We consider the random variables $W_{t}^{i}, i=1, \ldots, m$, defined by

$$
W_{t}^{i}=\int_{0}^{t} 1_{\left\{X_{u} \in B_{i}\right\}} d u
$$

The joint distribution of the $W_{t}^{i}$ and $X_{t}$ is given in the next theorem.

Theorem 4.3 For every $i, j \in S$, for every $t>0$ and $s_{1}, \ldots, s_{m} \in[0, t)$ such that $s_{1}+s_{2}+$ $\cdots+s_{m}<t$, we have

$\mathbb{P}\left\{W_{t}^{1} \leq s_{1}, \ldots, W_{t}^{m} \leq s_{m}, X_{t}=j \mid X_{0}=i\right\}=$

$$
\sum_{n=0}^{\infty} e^{-\lambda t} \frac{(\lambda t)^{n}}{n !} \sum_{\substack{k_{1} \geq 0, k_{2} \geq 0, \ldots, k_{m} \geq 0, k_{1}+k_{2}+\cdots+k_{m} \leq n}} n ! \theta_{n ; k_{1}, k_{2}, \ldots, k_{m}}^{t ; s_{1}, s_{2}, \ldots, s_{m}} \mathbb{P}\left\{V_{n}^{1} \leq k_{1}, \ldots, V_{n}^{m} \leq k_{m}, Z_{n}=j \mid Z_{0}=i\right\}
$$

where

$$
\theta_{n ; k_{1}, k_{2}, \ldots, k_{m}}^{t ; s_{1}, s_{2}, \ldots, s_{m}}=\frac{\left(\frac{s_{1}}{t}\right)^{k_{1}}\left(\frac{s_{2}}{t}\right)^{k_{2}} \cdots\left(\frac{s_{k}}{t}\right)^{k_{m}}\left(1-\frac{s_{1}+s_{2}+\cdots+s_{m}}{t}\right)^{n-\left(k_{1}+k_{2} \cdots+k_{m}\right)}}{k_{1} ! k_{2} ! \cdots k_{m} !\left(n-\left(k_{1}+k_{2} \cdots+k_{m}\right)\right) !} .
$$

Proof. We denote by $\widehat{W_{t}}, \widehat{V_{n}}, \widehat{s}$ and $\widehat{l}$ the vectors $\left(W_{t}^{1}, \ldots, W_{t}^{m}\right),\left(V_{n}^{1}, \ldots, V_{n}^{m}\right)$, $\left(s_{1}, \ldots, s_{m}\right)$ and $\left(l_{1}, \ldots, l_{m}\right)$ respectively. An inequality between two such vectors mean$\mathrm{s}$ the inequality for all their entries. For $n \geq 0$ we define the set $E_{n}$ as

$$
E_{n}=\left\{\widehat{l}=\left(l_{1}, l_{2}, \ldots, l_{m}\right) \in \mathbb{N}^{n} \mid l_{1}+l_{2}+\cdots+l_{m} \leq n\right\} .
$$

We then have

$$
\begin{aligned}
& \mathbb{P}\left\{\widehat{W_{t}} \leq \widehat{s}, X_{t}=j \mid X_{0}=i\right\} \\
& =\sum_{n=0}^{\infty} \mathbb{P}_{i}\left\{\widehat{W_{t}} \leq \widehat{s}, N_{t}=n, X_{t}=j\right\}
\end{aligned}
$$




$$
\begin{aligned}
& =\sum_{n=0}^{\infty} \mathbb{P}_{i}\left\{\widehat{W_{t}} \leq \widehat{s}, N_{t}=n, Z_{n}=j\right\} \quad \text { since }\left\{X_{t}\right\} \text { and }\left\{Z_{N_{t}}\right\} \text { are equivalent } \\
& =\sum_{n=0}^{\infty} \mathbb{P}_{i}\left\{N_{t}=n\right\} \mathbb{P}_{i}\left\{\widehat{W_{t}} \leq \widehat{s}, Z_{n}=j \mid N_{t}=n\right\} \\
& =\sum_{n=0}^{\infty} \mathbb{P}\left\{N_{t}=n\right\} \mathbb{P}_{i}\left\{\widehat{W_{t}} \leq \widehat{s}, Z_{n}=j \mid N_{t}=n\right\} \\
& =\sum_{n=0}^{\infty} \mathbb{P}\left\{N_{t}=n\right\} \sum_{\widehat{l} \in E_{n}} \mathbb{P}_{i}\left\{\widehat{W_{t}} \leq \widehat{s}, \widehat{V_{n}}=\widehat{l}, Z_{n}=j \mid N_{t}=n\right\} \\
& =\sum_{n=0}^{\infty} \mathbb{P}\left\{N_{t}=n\right\} \sum_{\widehat{l} \in E_{n}} \mathbb{P}_{i}\left\{\widehat{V_{n}}=\widehat{l}, Z_{n}=j \mid N_{t}=n\right\} \mathbb{P}_{i}\left\{\widehat{W_{t}} \leq \widehat{s} \mid \widehat{V_{n}}=\widehat{l}, Z_{n}=j, N_{t}=n\right\} \\
& =\sum_{n=0}^{\infty} \mathbb{P}\left\{N_{t}=n\right\} \sum_{\widehat{l} \in E_{n}} \mathbb{P}_{i}\left\{\widehat{V_{n}}=\widehat{l}, Z_{n}=j\right\} \mathbb{P}_{i}\left\{\widehat{W_{t}} \leq \widehat{s} \mid \widehat{V_{n}}=\widehat{l}, Z_{n}=j, N_{t}=n\right\}
\end{aligned}
$$

Note that the 4th and the last equalities follow from the independence of the processes $\left\{Z_{n}\right\}$ and $\left\{N_{t}\right\}$ and the fact that $X_{0}=Z_{0}$. Note also that in the 5 th equality, the summation over $\hat{l}$ should be for $\widehat{l} \in E_{n+1}$, but it can be done for $\widehat{l} \in E_{n}$ because if $l_{1}+l_{2}+\cdots+l_{m}=n+1$, we obtain that $\widehat{V_{n}}=\widehat{l}$ and $N_{t}=n$ imply that $V_{n}^{1}+\cdots+V_{n}^{m}=n+1$ and so that $W_{t}^{1}+\cdots+W_{t}^{m}=t$, which gives us $\mathbb{P}\left\{\widehat{W_{t}} \leq \widehat{s} \mid \widehat{V_{n}}=\widehat{l}, N_{t}=n\right\}=0$ since we have supposed that $s_{1}+\cdots+s_{m}<t$.

Let us consider now the expression $\mathbb{P}_{i}\left\{\widehat{W_{t}} \leq \widehat{s} \mid \widehat{V_{n}}=\widehat{l}, Z_{n}=j, N_{t}=n\right\}$.

For $\hat{l}=\left(l_{1}, l_{2}, \ldots, l_{m}\right) \in E_{n}$ and $i, j \in S$, we define the set

$$
G_{\widehat{l}, n}^{i, j}=\left\{\begin{array}{l|l}
\widehat{z}=\left(i, z_{1}, \ldots, z_{n-1}, j\right) \in S^{n+1} & \begin{array}{l}
l_{1} \text { entries of } \widehat{z} \text { are in } B_{1}, \\
l_{2} \text { entries of } \widehat{z} \text { are in } B_{2}, \\
\ldots, \\
l_{m} \text { entries of } \widehat{z} \text { are in } B_{m} \text { and } \\
n+1-\left(l_{1}+l_{2}+\cdots+l_{m}\right) \text { are in } B_{0}
\end{array}
\end{array}\right\}
$$

and we denote by $\widehat{Z}$ the random vector $\left(Z_{0}, \ldots, Z_{n}\right)$. We then have

$$
\begin{aligned}
& \mathbb{P}_{i}\left\{\widehat{W_{t}} \leq \widehat{s} \mid \widehat{V_{n}}=\widehat{l}, Z_{n}=j, N_{t}=n\right\} \\
& \quad=\sum_{\widehat{z} \in G_{\widehat{l}, n}^{i, j}} \mathbb{P}_{i}\left\{\widehat{W_{t}} \leq \widehat{s} \mid \widehat{Z}=\widehat{z}, \widehat{V_{n}}=\widehat{l}, Z_{n}=j, N_{t}=n\right\} \mathbb{P}_{i}\left\{\widehat{Z}=\widehat{z} \mid \widehat{V_{n}}=\widehat{l}, Z_{n}=j, N_{t}=n\right\}
\end{aligned}
$$




$$
\begin{aligned}
& =\sum_{\widehat{z} \in G_{\widehat{l, n}}^{i, j}} \mathbb{P}\left\{\widehat{W_{t}} \leq \widehat{s} \mid \widehat{Z}=\widehat{z}, \widehat{V_{n}}=\widehat{l}, N_{t}=n\right\} \mathbb{P}\left\{\widehat{Z}=\widehat{z} \mid \widehat{V_{n}}=\widehat{l}, Z_{n}=j, Z_{0}=i, N_{t}=n\right\} \\
& =\sum_{\widehat{z} \in G_{\widehat{l, n}}^{i, j}} \mathbb{P}\left\{\widehat{W_{t}} \leq \widehat{s} \mid \widehat{Z}=\widehat{z}, \widehat{V_{n}}=\widehat{l}, N_{t}=n\right\} \mathbb{P}\left\{\widehat{Z}=\widehat{z} \mid \widehat{V_{n}}=\widehat{l}, Z_{n}=j, Z_{0}=i\right\}
\end{aligned}
$$

where the last equality follows from the independence of the processes $\left\{Z_{n}\right\}$ and $\left\{N_{t}\right\}$.

We have

$$
\mathbb{P}\left\{\widehat{W_{t}} \leq \widehat{s} \mid \widehat{Z}=\widehat{z}, \widehat{V_{n}}=\widehat{l}, N_{t}=n\right\}=\mathbb{P}\left\{\widehat{T}(\widehat{l}) \leq \widehat{s} \mid \widehat{Z}=\widehat{z}, \widehat{V_{n}}=\widehat{l}, N_{t}=n\right\}
$$

where

$$
\widehat{T}(\widehat{l})=\left(\sum_{j=1}^{l_{1}} T_{i_{j}}, \sum_{j=l_{1}+1}^{l_{1}+l_{2}} T_{i_{j}}, \ldots, \sum_{j=l_{1}+l_{2}+\cdots+l_{m-1}+1}^{l_{1}+l_{2}+\cdots+l_{m}} T_{i_{j}}\right)
$$

So, using again the independence between $\left\{Z_{n}\right\}$ and $\left\{N_{t}\right\}$ and relation (3), we get

$$
\begin{aligned}
& \mathbb{P}\left\{\widehat{T}(\widehat{l}) \leq \widehat{s} \mid \widehat{Z}=\widehat{z}, \widehat{V_{n}}=\widehat{l}, N_{t}=n\right\}=\mathbb{P}\left\{\widehat{T}(\widehat{l}) \leq \widehat{s} \mid N_{t}=n\right\} \\
& =\sum_{\substack{k_{1} \geq l_{1}, k_{2} \geq l_{2}, \ldots, k_{m} \geq l_{m} \\
k_{1}+k_{2}+\cdots+k_{m} \leq n}} n ! \theta_{\substack{t ; k_{1}, k_{2}, \ldots, k_{m} \\
t ; s_{1}, s_{2}, \ldots, s_{m}}},
\end{aligned}
$$

Note that if one of the $l_{i}$ 's is equal to 0 , the corresponding entry of the vector $\widehat{T}(\widehat{l})$ becomes 0 and the preceding formula is still valid. Indeed, suppose for simplicity that $l_{m}=0$. We obtain

$$
\begin{aligned}
& \sum_{\substack{k_{1} \geq l_{1}, \ldots, k_{m} \geq 0, k_{1}+\cdots+k_{m} \leq n}} n ! \theta_{n ; k_{1}, k_{2}, \ldots, k_{m}}^{t ; s_{1}, s_{2}, \ldots, s_{m}}=\sum_{\substack{k_{1} \geq l_{1}, \ldots, k_{m-1} \geq l_{m-1}, k_{1}+\cdots+k_{m-1} \leq n}} \sum_{k_{m}=0}^{n-\left(k_{1}+\cdots+k_{m-1}\right)} n ! \theta_{n ; k_{1}, \ldots, k_{m}}^{t ; s_{1}, \ldots, s_{m}} \\
& =\sum_{\substack{k_{1} \geq l_{1}, \ldots, k_{m-1} \geq l_{m-1} \\
k_{1}+\cdots+k_{m-1} \leq n}} n ! \theta_{n ; k_{1}, \ldots, k_{m-1}}^{t ; s_{1}, \ldots, s_{m-1}} \sum_{k_{m}=0}^{n-\left(k_{1}+\cdots+k_{m-1}\right)} \frac{\left(n-\left(k_{1}+\cdots+k_{m-1}\right)\right) ! \theta_{n-\left(k_{1}+\cdots+k_{m-1}\right) ; k_{m}}^{t ; s_{m}}}{\left(1-\frac{s_{1}+\cdots+s_{m-1}}{t}\right)^{n-\left(k_{1}+\cdots+k_{m-1}\right)}} \\
& =\sum_{k_{1} \geq l_{1}, \ldots, k_{m-1} \geq l_{m-1},} n ! \theta_{n ; k_{1}, \ldots, k_{m-1}}^{t ; s_{1}, \ldots, s_{m-1}} . \\
& k_{1}+\cdots+k_{m-1} \leq n
\end{aligned}
$$


Note also that if all the $l_{i}$ 's are equal to 0 , then all the entries of $\widehat{T}(\widehat{l})$ are equal to 0 and the formula is still valid since

$$
\sum_{\substack{k_{1} \geq 0, k_{2} \geq 0, \ldots, k_{m} \geq 0 \\ k_{1}+k_{2}+\cdots+k_{m} \leq n}} n ! \theta_{n ; k_{1}, k_{2}, \ldots, k_{m}}^{t ; s_{1}, s_{2}, \ldots, s_{m}}=1 .
$$

Putting together these results, we obtain

$$
\begin{aligned}
& \mathbb{P}_{i}\left\{\widehat{W_{t}} \leq \widehat{s} \mid \widehat{V_{n}}=\widehat{l}, Z_{n}=j, N_{t}=n\right\}
\end{aligned}
$$

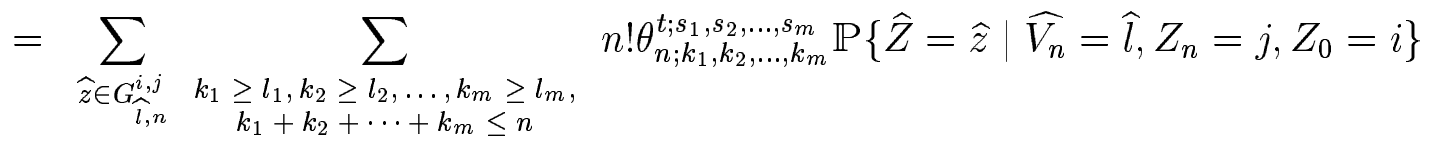

$$
\begin{aligned}
& =\sum_{\substack{k_{1} \geq l_{1}, k_{2} \geq l_{2}, \ldots, k_{m} \geq l_{m} \\
k_{1}+k_{2}+\cdots+k_{m} \leq n}} n ! \theta_{n ; k_{1}, k_{2}, \ldots, k_{m}}^{t ; s_{1}, s_{2}, \ldots, s_{m}} \sum_{\widehat{z} \in G_{\widehat{l}, n}^{i, j}} \mathbb{P}\left\{\widehat{Z}=\widehat{z} \mid \widehat{V_{n}}=\widehat{l}, Z_{n}=j, Z_{0}=i\right\} \\
& =\sum_{\substack{k_{1} \geq l_{1}, k_{2} \geq l_{2}, \ldots, k_{m} \geq l_{m} \\
k_{1}+k_{2}+\cdots+k_{m} \leq n}} n ! \theta_{n ; k_{1}, k_{2}, \ldots, k_{m}}^{t ; s_{1}, s_{2}, \ldots, s_{m}}
\end{aligned}
$$

and so,

$$
\begin{aligned}
& \mathbb{P}\left\{\widehat{W_{t}} \leq \widehat{s}, X_{t}=j \mid X_{0}=i\right\} \\
& =\sum_{n=0}^{\infty} e^{-\lambda t} \frac{(\lambda t)^{n}}{n !} \sum_{\substack{l \in E_{n} \\
k_{1} \geq l_{1}, k_{2} \geq l_{2}, \ldots, k_{m} \geq l_{m}, k_{1}+k_{2}+\cdots+k_{m} \leq n}} n ! \theta_{n ; k_{1}, k_{2}, \ldots, k_{m}}^{t ; s_{1}, s_{2}, \ldots, s_{m}} \mathbb{P}\left\{\widehat{V_{n}}=\widehat{l}, Z_{n}=j \mid Z_{0}=i\right\} \\
& =\sum_{n=0}^{\infty} e^{-\lambda t} \frac{(\lambda t)^{n}}{n !} \sum_{\widehat{k} \in E_{n}} n ! \theta_{\substack{n ; k_{1}, k_{2}, \ldots, k_{m} \\
t ; s_{1}, s_{2}, \ldots, s_{m}}} \sum_{\substack{l_{1} \geq k_{1}, l_{2} \geq k_{2}, \ldots, l_{m} \geq k_{m} \\
l_{1}+l_{2}+\cdots+l_{m} \leq n}} \mathbb{P}\left\{\widehat{V_{n}}=\widehat{l}, Z_{n}=j \mid Z_{0}=i\right\} \\
& =\sum_{n=0}^{\infty} e^{-\lambda t} \frac{(\lambda t)^{n}}{n !} \sum_{\widehat{k} \in E_{n}} n ! \theta_{\substack{n_{1}, k_{1}, k_{2}, \ldots, k_{m} \\
t ; s_{1}, s_{2}, \ldots, s_{m}}} \mathbb{P}\left\{\widehat{V_{n}} \leq \widehat{l}\right\} \\
& =\sum_{n=0}^{\infty} e^{-\lambda t} \frac{(\lambda t)^{n}}{n !} \sum_{\substack{k_{1} \geq 0, k_{2} \geq 0, \ldots, k_{m} \geq 0, k_{1}+k_{2}+\cdots+k_{m} \leq n}} n ! \theta_{n ; k_{1}, k_{2}, \ldots, k_{m}}^{t ; s_{1}, s_{2}, \ldots, s_{m}} \mathbb{P}\left\{\widehat{V_{n}} \leq \widehat{l}, Z_{n}=j \mid Z_{0}=i\right\} .
\end{aligned}
$$

$\mathrm{RR} \mathrm{n}^{\circ} 3806$ 
Thus, the proof is completed.

From relation (9), for $t>0$, the distribution $\mathbb{P}\left\{W_{t}^{1} \leq s_{1}, \ldots, W_{t}^{m} \leq s_{m}, X_{t}=j \mid X_{0}=\right.$ $i$ \} is differentiable with respect to $t$ and also with respect to $s_{1}, s_{2}, \ldots, s_{m}$ for $s_{1}, \ldots, s_{m} \in$ $(0, t)$ and $s_{1}+\cdots+s_{m} \in(0, t)$. These derivatives are given by the following corollary.

Corollary 4.4 For $i, j \in S$, for $t>0$ and $s_{1}, \ldots, s_{m} \in(0, t)$ such that $s_{1}+\cdots+s_{m}<t$, we have

$$
\begin{aligned}
& \frac{\partial \mathbb{P}\left\{W_{t}^{1} \leq s_{1}, \ldots, W_{t}^{m} \leq s_{m}, X_{t}=j \mid X_{0}=i\right\}}{\partial s_{1}}= \\
& \lambda \sum_{n=0}^{\infty} e^{-\lambda t} \frac{(\lambda t)^{n}}{n !} \sum_{\substack{k_{1} \geq 0, \ldots, k_{m} \geq 0, k_{1}+\cdots+k_{m} \leq n}} n ! \theta_{n ; k_{1}, k_{2}, \ldots, k_{m}}^{t ; s_{1}, s_{2}, \ldots, s_{m}} \\
& \mathbb{P}_{i}\left\{V_{n+1}^{1}=k_{1}+1, V_{n+1}^{2} \leq k_{2}, \ldots, V_{n+1}^{m} \leq k_{m}, Z_{n+1}=j\right\}
\end{aligned}
$$

and

$$
\begin{aligned}
& \frac{\partial^{m} \mathbb{P}\left\{W_{t}^{1} \leq s_{1}, \ldots, W_{t}^{m} \leq s_{m}, X_{t}=j \mid X_{0}=i\right\}}{\partial s_{1} \partial s_{2} \cdots \partial s_{m}}= \\
& \lambda^{m} \sum_{n=0}^{\infty} e^{-\lambda t} \frac{(\lambda t)^{n}}{n !} \sum_{\substack{k_{1} \geq 0, \ldots, k_{m} \geq 0, k_{1}+\cdots+k_{m} \leq n}} n ! \theta_{n ; k_{1}, k_{2}, \ldots, k_{m}}^{t ; s_{1}, s_{2}, \ldots, s_{m}} \\
& \mathbb{P}_{i}\left\{V_{n+m}^{1}=k_{1}+1, \ldots, V_{n+m}^{m}=k_{m}+1, Z_{n+m}=j\right\}
\end{aligned}
$$

and

$$
\begin{aligned}
& \frac{\partial \mathbb{P}\left\{W_{t}^{1} \leq s_{1}, \ldots, W_{t}^{m} \leq s_{m}, X_{t}=j \mid X_{0}=i\right\}}{\partial t}= \\
& \lambda \sum_{n=0}^{\infty} e^{-\lambda t} \frac{(\lambda t)^{n}}{n !} \sum_{\substack{k_{1} \geq 0, \ldots, k_{m} \geq 0 \\
k_{1}+\cdots+k_{m} \leq n}} n ! \theta_{n ; k_{1}, k_{2}, \ldots, k_{m}}^{t ; s_{1}, s_{2}, \ldots, s_{m}}\left[\begin{array}{r}
\mathbb{P}_{i}\{ \\
\left\{V_{n+1}^{1} \leq k_{1}, \ldots, V_{n+1}^{m} \leq k_{m}, Z_{n+1}=j\right\} \\
\left.-\mathbb{P}_{i}\left\{V_{n}^{1} \leq k_{1}, \ldots, V_{n}^{m} \leq k_{m}, Z_{n}=j\right\}\right]
\end{array}\right.
\end{aligned}
$$

Proof. In order to simplify notation, we define

$$
F_{i, j}\left(n, k_{1}, k_{2}, \ldots, k_{m}\right)=\mathbb{P}\left\{V_{n}^{1} \leq k_{1}, V_{n}^{2} \leq k_{2}, \ldots, V_{n}^{m} \leq k_{m}, Z_{n}=j \mid Z_{0}=i\right\},
$$


and

$$
f_{i, j}\left(n, k_{1}, k_{2}, \ldots, k_{m}\right)=\mathbb{P}\left\{V_{n}^{1}=k_{1}, V_{n}^{2} \leq k_{2}, \ldots, V_{n}^{m} \leq k_{m}, Z_{n}=j \mid Z_{0}=i\right\} .
$$

We thus have the relation

$$
F_{i, j}\left(n, k_{1}+1, k_{2}, \ldots, k_{m}\right)-F_{i, j}\left(n, k_{1}, k_{2}, \ldots, k_{m}\right)=f_{i, j}\left(n, k_{1}+1, k_{2}, \ldots, k_{m}\right) .
$$

For $t>0$ and $s_{1}, \ldots, s_{m} \in(0, t)$ such that $s_{1}+\cdots+s_{m}<t$, we have from relation (9)

$$
\begin{aligned}
& \frac{\partial \mathbb{P}\left\{W_{t}^{1} \leq s_{1}, \ldots, W_{t}^{m} \leq s_{m}, X_{t}=j \mid X_{0}=i\right\}}{\partial s_{1}} \\
& =\frac{1}{t} \sum_{n=1}^{\infty} e^{-\lambda t} \frac{(\lambda t)^{n}}{n !} \sum_{\substack{k_{1} \geq 1, k_{2} \geq 0, \ldots, k_{m} \geq 0, k_{1}+\cdots+k_{m} \leq n}} n ! \theta_{n ; k_{1}-1, k_{2}, \ldots, k_{m}}^{t ; s_{1}, s_{2}, \ldots, s_{m}} F_{i, j}\left(n, k_{1}, k_{2}, \ldots, k_{m}\right) \\
& -\frac{1}{t} \sum_{n=1}^{\infty} e^{-\lambda t} \frac{(\lambda t)^{n}}{n !} \sum_{\substack{k_{1} \geq 0, k_{2} \geq 0, \ldots, k_{m} \geq 0, k_{1}+\cdots+k_{m} \leq n-1}} n ! \theta_{n-1 ; k_{1}, \ldots, k_{m}}^{t ; s_{1}, \ldots, s_{m}} F_{i, j}\left(n, k_{1}, k_{2}, \ldots, k_{m}\right) \\
& =\frac{1}{t} \sum_{n=1}^{\infty} e^{-\lambda t} \frac{(\lambda t)^{n}}{n !} \sum_{\substack{k_{1} \geq 0, \ldots, k_{m} \geq 0, k_{1}+\cdots+k_{m} \leq n-1}} n ! \theta_{n-1 ; k_{1}, \ldots, k_{m}}^{t ; s_{1}, \ldots, s_{m}} F_{i, j}\left(n, k_{1}+1, k_{2}, \ldots, k_{m}\right) \\
& -\frac{1}{t} \sum_{n=1}^{\infty} e^{-\lambda t} \frac{(\lambda t)^{n}}{n !} \sum_{\substack{k_{1} \geq 0, \ldots, k_{m} \geq 0, k_{1}+\ldots+k_{m} \leq n-1}} n ! \theta_{n-1 ; k_{1}, \ldots, k_{m}}^{t ; s_{1}, \ldots, s_{m}} F_{i, j}\left(n, k_{1}, k_{2}, \ldots, k_{m}\right) \\
& =\frac{1}{t} \sum_{n=1}^{\infty} e^{-\lambda t} \frac{(\lambda t)^{n}}{n !} \sum_{\substack{k_{1} \geq 0, \ldots, k_{m} \geq 0, k_{1}+\cdots+k_{m} \leq n-1}} n ! \theta_{n-1 ; k_{1}, \ldots, k_{m}}^{t ; s_{1}, \ldots, s_{m}} f_{i, j}\left(n, k_{1}+1, k_{2}, \ldots, k_{m}\right) \\
& =\lambda \sum_{n=0}^{\infty} e^{-\lambda t} \frac{(\lambda t)^{n}}{n !} \sum_{\substack{k_{1} \geq 0, \ldots, k_{m} \geq 0, k_{1}+\cdots+k_{m} \leq n}} n ! \theta_{n ; k_{1}, \ldots, k_{m}}^{t ; s_{1}, \ldots, s_{m}} f_{i, j}\left(n+1, k_{1}+1, k_{2}, \ldots, k_{m}\right) .
\end{aligned}
$$

The second relation follows easily using the same argument. For the third relation, since $t>0$, we write

$\mathrm{RR} \mathrm{n}^{\circ} 3806$ 
$\mathbb{P}\left\{W_{t}^{1} \leq s_{1}, \ldots, W_{t}^{m} \leq s_{m}, X_{t}=j \mid X_{0}=i\right\}$

$$
=e^{-\lambda t} \sum_{n=0}^{\infty} \lambda^{n} \sum_{\substack{k_{1} \geq 0, \ldots, k_{m} \geq 0 \\ k_{1}+\cdots+k_{m} \leq n}} t^{n} \theta_{n ; k_{1}, \ldots, k_{m}}^{t ; s_{1}, \ldots, s_{m}} F_{i, j}\left(n, k_{1}, k_{2}, \ldots, k_{m}\right)
$$

and so,

$$
\begin{aligned}
& \frac{\partial \mathbb{P}\left\{W_{t}^{1} \leq s_{1}, \ldots, W_{t}^{m} \leq s_{m}, X_{t}=j \mid X_{0}=i\right\}}{\partial t} \\
& =-\lambda e^{-\lambda t} \sum_{n=0}^{\infty} \lambda^{n} \sum_{\substack{k_{1} \geq 0, \ldots, k_{m} \geq 0, k_{1}+\cdots+k_{m} \leq n}} t^{n} \theta_{n ; k_{1}, \ldots, k_{m}}^{t ; s_{1}, \ldots, s_{m}} F_{i, j}\left(n, k_{1}, k_{2}, \ldots, k_{m}\right) \\
& +e^{-\lambda t} \sum_{n=1}^{\infty} \lambda^{n} \sum_{\substack{k_{1} \geq 0, \ldots, k_{m} \geq 0, k_{1}+\cdots+k_{m} \leq n-1}} t^{n-1} \theta_{n-1 ; k_{1}, \ldots, k_{m}}^{t ; s_{1}, \ldots, s_{m}} F_{i, j}\left(n, k_{1}, k_{2}, \ldots, k_{m}\right) \\
& =-\lambda e^{-\lambda t} \sum_{n=0}^{\infty} \lambda^{n} \sum_{\substack{k_{1} \geq 0, \ldots, k_{m} \geq 0, k_{1}+\ldots+k_{m} \leq n}} t^{n} \theta_{n ; k_{1}, \ldots, k_{m}}^{t ; s_{1}, \ldots, s_{m}} F_{i, j}\left(n, k_{1}, k_{2}, \ldots, k_{m}\right) \\
& +e^{-\lambda t} \sum_{n=0}^{\infty} \lambda^{n+1} \sum_{\substack{k_{1} \geq 0, \ldots, k_{m} \geq 0, k_{1}+\cdots+k_{m} \leq n}} t^{n} \theta_{n ; k_{1}, \ldots, k_{m}}^{t ; s_{1}, \ldots, s_{m}} F_{i, j}\left(n+1, k_{1}, k_{2}, \ldots, k_{m}\right) \\
& =\lambda \sum_{n=0}^{\infty} e^{-\lambda t} \frac{(\lambda t)^{n}}{n !} \sum_{\substack{k_{1} \geq 0, \ldots, k_{m} \geq 0 \\
k_{1}+\cdots+k_{m} \leq n}} n ! \theta_{n ; k_{1}, \ldots, k_{m}}^{t ; s_{1}, \ldots, s_{m}}\left[F_{i, j}\left(n+1, k_{1}, k_{2}, \ldots, k_{m}\right)-F_{i, j}\left(n, k_{1}, k_{2}, \ldots, k_{m}\right)\right]
\end{aligned}
$$

Note that if $s_{1}+s_{2}+\cdots+s_{m} \geq t$, then we trivially have

$$
\mathbb{P}\left\{W_{t}^{1}>s_{1}, \ldots, W_{t}^{m}>s_{m}, X_{t}=j \mid X_{0}=i\right\}=0
$$

so relation (8) applies by replacing the $V_{n}^{l}$ and the $k_{l}$ by the $W_{t}^{l}$ and the $s_{l}$ respectively. 


\section{Distribution of Weighted Sums of Occupation Times}

A constant performance level or reward rate $\rho(i)$ is associated with each state $i$ of $S$ and we consider the random variable $Y_{t}$ defined by

$$
Y_{t}=\int_{0}^{t} \rho\left(X_{u}\right) d u
$$

We denote by $m+1$ the number of distinct rewards and we denote their values by

$$
r_{0}<r_{1}<\cdots<r_{m-1}<r_{m}
$$

We then have $Y_{t} \in\left[r_{0} t, r_{m} t\right]$ with probability one. Without loss of generality, we suppose that $r_{0}=0$. This can be easily done by considering the random variable $Y_{t}-r_{0} t$ instead of $Y_{t}$ and the reward rates $r_{i}-r_{0}$ instead of $r_{i}$.

The state space $S$ can be then divided into disjoint subsets $B_{m}, B_{m-1}, \ldots, B_{0}$ where $B_{l}$ is composed by the states of $S$ having as reward rate $r_{l}$, that is $B_{l}=\left\{i \in S / \rho(i)=r_{l}\right\}$.

With this notation, we have

$$
Y_{t}=\sum_{l=1}^{m} r_{l} \int_{0}^{t} 1_{\left\{X_{u} \in B_{l}\right\}} d u=\sum_{l=1}^{m} r_{l} W_{t}^{l} .
$$

Since the distribution of each $W_{t}^{l}$ has at most two jumps at points 0 and $t$, we conclude that the distribution of $Y_{t}$ has at most $m+1$ jumps at points $r_{0} t=0, r_{1} t, \ldots, r_{m} t$. For $t>0$, the jump at point $x=r_{l} t$ is equal to the probability that the process $X$, starting in subset $B_{l}$, stays during the whole interval $[0, t)$ in the subset $B_{l}$, that is

$$
\mathbb{P}\left\{Y_{t}=r_{l} t\right\}=\alpha_{B_{l}} e^{A_{B_{l} B_{l}} t} \mathbb{B}_{B_{l}} \text { for } t>0,
$$

where $\mathbb{1}_{B_{l}}$ is the column vector of dimension $\left|B_{l}\right|$ with all entries equal to 1 . For every $i, j \in S$ and $t>0$, we define the functions $F_{i, j}(t, x)$ by

$$
F_{i, j}(t, x)=\mathbb{P}\left\{Y_{t}>x, X_{t}=j \mid X_{0}=i\right\},
$$

and we denote by $F(t, x)$ the matrix containing the terms $F_{i, j}(t, x)$ for $i, j \in S$. The matrices $A, P$ and $F(t, x)$ can then be written using the partition $B_{m}, B_{m-1}, \ldots, B_{0}$ of $S$ as

$$
A=\left(A_{B_{u} B_{v}}\right)_{0 \leq u, v \leq m} ; P=\left(P_{B_{u} B_{v}}\right)_{0 \leq u, v \leq m} ; F(t, x)=\left(F_{B_{u} B_{v}}(t, x)\right)_{0 \leq u, v \leq m} .
$$

$\mathrm{RR} \mathrm{n}^{\circ} 3806$ 
Note that we then have, for $t>0$ and $l=0,1, \ldots, m$,

$$
\mathbb{P}\left\{Y_{t}=r_{l} t, X_{t}=j \mid X_{0}=i\right\}=\left\{\begin{array}{cl}
\left(e^{A_{B_{l} B_{l}}}\right)_{i, j} & \text { if } i, j \in B_{l} \\
0 & \text { otherwise }
\end{array},\right.
$$

that is

$$
\mathbb{P}\left\{Y_{t}=r_{l} t, X_{t}=j \mid X_{0}=i\right\}=\sum_{n=0}^{\infty} e^{-\lambda t} \frac{(\lambda t)^{n}}{n !}\left(P_{B_{l} B_{l}}^{n}\right)_{i, j} 1_{\left\{i, j \in B_{l}\right\}} .
$$

The distribution $F_{i, j}(t, x)$ can be obtained from relation (13), using the joint distribution of the $W_{t}^{l}$ obtained in the previous section. From corollary $4.4, F_{i, j}(t, x)$ is differentiable with respect to $x$ and $t$ in the domain

$$
E=\left\{(t, x) ; t>0 \text { and } x \in \bigcup_{l=1}^{m}\left(r_{l-1} t, r_{l} t\right)\right\} .
$$

The initial conditions are given, for $t>0$, by

$$
F_{i, j}(t, 0)=\mathbb{P}\left\{X_{t}=j \mid X_{0}=i\right\}-\mathbb{P}\left\{Y_{t}=0, X_{t}=j \mid X_{0}=i\right\},
$$

that is, in matrix notation,

$$
F_{B_{u} B_{v}}(t, 0)=\left(e^{A t}\right)_{B_{u} B_{v}}-e^{A_{B_{0} B_{0}} t} 1_{\{u=v=0\}}
$$

which can also be written as

$$
F_{B_{u} B_{v}}(t, 0)=\sum_{n=0}^{\infty} e^{-\lambda t} \frac{(\lambda t)^{n}}{n !}\left[\left(P^{n}\right)_{B_{u} B_{v}}-P_{B_{0} B_{0}}^{n} 1_{\{u=v=0\}}\right] .
$$

\subsection{Backward and Forward Equations}

In the following, we derive the backward and forward equations satisfied by the distribution of the couple $\left(Y_{t}, X_{t}\right)$ and we give expressions of the solution of these equations.

Let us first state some useful results in the following lemma. Recall that $\{N(t)\}$ is a Poisson process with rate $\lambda$ and that it is independent of the uniformized Markov chain $Z$. We denote by $N(t, t+s)$ the number of transitions during the interval $[t, t+s)$.

\section{Lemma 5.1}

$$
\begin{gathered}
\mathbb{P}\left\{N(t, t+s)=0 \mid X_{t}=j\right\}=e^{-\lambda s} \\
\mathbb{P}\left\{X_{t+s}=j, N(t, t+s)=1 \mid X_{t}=i\right\}=P_{i, j} \lambda s e^{-\lambda s} \\
\mathbb{P}\left\{N(t, t+s) \geq 2 \mid X_{0}=i\right\}=o(s) .
\end{gathered}
$$


Proof. Recall that the processes $\left\{X_{t}\right\}$ and $\left\{Z_{N(t)}\right\}$ are equivalent and $Z_{0}=X_{0}$. For relation (16), by homogeneity, we have

$$
\begin{aligned}
\mathbb{P}\left\{N(t, t+s)=0 \mid X_{t}=j\right\} & =\mathbb{P}\left\{N(s)=0 \mid X_{0}=j\right\} \\
& =\mathbb{P}\left\{N(s)=0 \mid Z_{0}=j\right\} \\
& =\mathbb{P}\{N(s)=0\} \\
& =e^{-\lambda s}
\end{aligned}
$$

In the same way, for relation (17), we have

$$
\begin{aligned}
\mathbb{P}\left\{X_{t+s}=j, N(t, t+s)=1 \mid X_{t}=i\right\} & =\mathbb{P}\left\{X_{s}=j, N(s)=1 \mid X_{0}=i\right\} \\
& =\mathbb{P}\left\{Z_{1}=j, N(s)=1 \mid Z_{0}=i\right\} \\
& =\mathbb{P}\left\{N(s)=1 \mid Z_{0}=i, Z_{1}=j\right\} P_{i, j} \\
& =\mathbb{P}\{N(s)=1\} P_{i, j} \\
& =P_{i, j} \lambda s e^{-\lambda s}
\end{aligned}
$$

For relation (18), we have

$$
\begin{aligned}
\mathbb{P}\left\{N(t, t+s) \geq 2 \mid X_{t}=i\right\} & =\mathbb{P}\left\{N(s) \geq 2 \mid X_{0}=i\right\} \\
& =\mathbb{P}\{N(s) \geq 2\} \\
& =1-e^{-\lambda s}-\lambda s e^{-\lambda s} \\
& =o(s)
\end{aligned}
$$

This completes the proof.

The following theorem states the forward equation describing the behavior of the pair $\left(Y_{t}, X_{t}\right)$.

Theorem 5.2 For $t>0, i, j \in S, 1 \leq h \leq m$ and $x \in\left(r_{h-1} t, r_{h} t\right)$ we have

$$
\frac{\partial F_{i, j}(t, x)}{\partial t}=-\rho(j) \frac{\partial F_{i, j}(t, x)}{\partial x}+\sum_{k \in S} F_{i, k}(t, x) A_{k, j} .
$$

Proof. By conditioning on the number of transitions in the interval $[t, t+s)$, we have

$$
\begin{aligned}
\mathbb{P}_{i}\left\{Y_{t+s}>x, X_{t+s}=j\right\}= & \mathbb{P}_{i}\left\{Y_{t+s}>x, X_{t+s}=j, N(t, t+s)=0\right\} \\
& +\mathbb{P}_{i}\left\{Y_{t+s}>x, X_{t+s}=j, N(t, t+s)=1\right\} \\
& +\mathbb{P}_{i}\left\{Y_{t+s}>x, X_{t+s}=j, N(t, t+s) \geq 2\right\}
\end{aligned}
$$

$\mathrm{RR} \mathrm{n}^{\circ} 3806$ 
We consider the three terms of the right hand side separately.

For the first term, since $X_{t+s}=j$ and $N(t, t+s)=0$ is equivalent to $X_{t}=j$ and $N(t, t+s)=0$, we have

$$
\begin{aligned}
& \mathbb{P}_{i}\left\{Y_{t+s}>x, X_{t+s}=j, N(t, t+s)=0\right\}=\mathbb{P}_{i}\left\{Y_{t+s}>x, X_{t}=j, N(t, t+s)=0\right\} \\
& \quad=\mathbb{P}_{i}\left\{Y_{t+s}>x \mid X_{t}=j, N(t, t+s)=0\right\} \mathbb{P}_{i}\left\{X_{t}=j, N(t, t+s)=0\right\} \\
& \quad=\mathbb{P}_{i}\left\{Y_{t}>x-\rho(j) s \mid X_{t}=j, N(t, t+s)=0\right\} \mathbb{P}_{i}\left\{X_{t}=j, N(t, t+s)=0\right\} \\
& \quad=\mathbb{P}_{i}\left\{Y_{t}>x-\rho(j) s \mid X_{t}=j\right\} \mathbb{P}_{i}\left\{X_{t}=j, N(t, t+s)=0\right\} \\
& \quad=\mathbb{P}_{i}\left\{Y_{t}>x-\rho(j) s, X_{t}=j\right\} \mathbb{P}_{i}\left\{N(t, t+s)=0 \mid X_{t}=j\right\} \\
& \quad=\mathbb{P}\left\{N(t, t+s)=0 \mid X_{t}=j\right\} F_{i, j}(t, x-\rho(j) s) \\
& \quad=e^{-\lambda s} F_{i, j}(t, x-\rho(j) s) \\
& \quad=(1-\lambda s) F_{i, j}(t, x-\rho(j) s)+o(s)
\end{aligned}
$$

where the second equality follows from the fact that if $X_{t}=j$ and $N(t, t+s)=0$ then we have $Y_{t+s}=Y_{t}+\int_{t}^{t+s} \rho\left(X_{u}\right) d u=Y_{t}+\rho(j) s$, the third one and the fifth one follow from the Markov property, and the sixth follows from relation (16).

For the second term that we denote by $G(s)$, we define

$$
G_{k}(s)=\mathbb{P}_{i}\left\{Y_{t+s}>x \mid X_{t}=k, X_{t+s}=j, N(t, t+s)=1\right\} .
$$

We then have

$$
\begin{aligned}
G(s) & =\mathbb{P}_{i}\left\{Y_{t+s}>x, X_{t+s}=j, N(t, t+s)=1\right\} \\
& =\sum_{k \in S} G_{k}(s) \mathbb{P}_{i}\left\{X_{t}=k, X_{t+s}=j, N(t, t+s)=1\right\}
\end{aligned}
$$

Let us define $\rho_{\min }=\min \left\{\rho_{i}\right\}$ and $\rho_{\max }=\max \left\{\rho_{i}\right\}$.

Since $Y_{t}+\rho_{\min } s \leq Y_{t+s} \leq Y_{t}+\rho_{\max } s$, we get

$$
\mathbb{P}_{i}\left\{Y_{t}>x-\rho_{\min } s \mid X_{t}=k, X_{t+s}=j, N(t, t+s)=1\right\} \leq G_{k}(s)
$$

and

$$
G_{k}(s) \leq \mathbb{P}_{i}\left\{Y_{t}>x-\rho_{\max } s \mid X_{t}=k, X_{t+s}=j, N(t, t+s)=1\right\}
$$


Using the Markov property,

$$
\mathbb{P}_{i}\left\{Y_{t}>x-\rho_{\min } s \mid X_{t}=k\right\} \leq G_{k}(s) \leq \mathbb{P}_{i}\left\{Y_{t}>x-\rho_{\max } s \mid X_{t}=k\right\},
$$

We thus obtain

$$
\sum_{k \in S} F_{i, k}\left(t, x-\rho_{\min } s\right) U_{k, j}(s) \leq G(s) \leq \sum_{k \in S} F_{i, k}\left(t, x-\rho_{\max } s\right) U_{k, j}(s),
$$

where $U_{k, j}(s)$ is defined by

$$
U_{k, j}(s)=\mathbb{P}\left\{X_{t+s}=j, N(t, t+s)=1 \mid X_{t}=k\right\} .
$$

From relation (17), we have

$$
\lim _{s \longrightarrow 0} \frac{U_{k, j}(s)}{s}=\lambda P_{k, j}
$$

so we obtain

$$
\lim _{s \rightarrow 0} \frac{G(s)}{s}=\lambda \sum_{k \in S} F_{i, k}(t, x) P_{k, j} .
$$

For the third term, we have from relation (18),

$$
\mathbb{P}_{i}\left\{Y_{t+s}>x, X_{t+s}=j, N(t, t+s) \geq 2\right\} \leq \mathbb{P}_{i}\{N(t, t+s) \geq 2\}=o(s) .
$$

Putting together the three terms, we obtain

$$
\begin{gathered}
\frac{F_{i, j}(t+s, x)-F_{i, j}(t, x)}{s}=\frac{(1-\lambda s) F_{i, j}(t, x-\rho(j) s)-F_{i, j}(t, x)}{s}+\frac{G(s)}{s}+\frac{o(s)}{s} \\
=\frac{F_{i, j}(t, x-\rho(j) s)-F_{i, j}(t, x)}{s}-\lambda F_{i, j}(t, x-\rho(j) s)+\frac{G(s)}{s}+\frac{o(s)}{s}
\end{gathered}
$$

If we let now $s$ tend to 0 , we get

$$
\frac{\partial F_{i, j}(t, x)}{\partial t}=-\rho(j) \frac{\partial F_{i, j}(t, x)}{\partial x}-\lambda F_{i, j}(t, x)+\lambda \sum_{k \in S} F_{i, k}(t, x) P_{k, j} .
$$

Since $P=I+A / \lambda$, we obtain

$$
\frac{\partial F_{i, j}(t, x)}{\partial t}=-\rho(j) \frac{\partial F_{i, j}(t, x)}{\partial x}+\sum_{k \in S} F_{i, k}(t, x) A_{k, j} .
$$

The proof is thus complete.

$\mathrm{RR} \mathrm{n}^{\circ} 3806$ 
Corollary 5.3 For $t>0,0 \leq p \leq m, i \in B_{p}, j \in S, 1 \leq h \leq m$ and $x \in\left(r_{h-1} t, r_{h} t\right)$ we have

$$
F_{i, j}(t, x)=\sum_{k \in S} \int_{0}^{t} F_{i, k}(t-u, x-\rho(j) u) \lambda e^{-\lambda u} d u P_{k, j}+e^{-\lambda t} 1_{\{h \leq p\}} 1_{\{i=j\}}
$$

Proof. Consider equation (19) and the functions $\varphi_{i, j}$ defined by

$$
\varphi_{i, j}(u)=F_{i, j}(t-u, x-\rho(j) u) e^{-\lambda u} .
$$

Differentiating with respect to $u$, we get

$$
\varphi_{i, j}^{\prime}(u)=e^{-\lambda u}\left[-\frac{\partial F_{i, j}}{\partial t}-\rho(j) \frac{\partial F_{i, j}}{\partial x}\right](t-u, x-\rho(j) u)-F_{i, j}(t-u, x-\rho(j) u) \lambda e^{-\lambda u} .
$$

This gives using equation (19) and the relation $A=-\lambda(I-P)$,

$$
\begin{aligned}
\varphi_{i, j}^{\prime}(u) & =-\sum_{k \in S} F_{i, k}(t-u, x-\rho(j) u) A_{k, j} e^{-\lambda u}-F_{i, j}(t-u, x-\rho(j) u) \lambda e^{-\lambda u} \\
& =-\sum_{k \in S} F_{i, k}(t-u, x-\rho(j) u) \lambda e^{-\lambda u} P_{k, j}
\end{aligned}
$$

Integrating now this expression between 0 and $t$, we obtain

$$
\varphi_{i, j}(t)-\varphi_{i, j}(0)=-\sum_{k \in S} \int_{0}^{t} F_{i, k}(t-u, x-\rho(j) u) \lambda e^{-\lambda u} d u P_{k, j} .
$$

Finally, we have $\varphi_{i, j}(0)=F_{i, j}(t, x)$ and

$$
\varphi_{i, j}(t)=F_{i, j}(0, x-\rho(j) t) e^{-\lambda t}=e^{-\lambda t} 1_{\{x-\rho(j) t<0\}} 1_{\{i=j\}}=e^{-\lambda t} 1_{\{h \leq p\}} 1_{\{i=j\}},
$$

which completes the proof.

We now derive the backward equation for the evolution of the pair $\left(Y_{t}, X_{t}\right)$.

Theorem 5.4 For $t>0,0 \leq p \leq m, i \in B_{p}, j \in S, 1 \leq h \leq m$ and $x \in\left(r_{h-1} t, r_{h} t\right)$, we have

$$
F_{i, j}(t, x)=\sum_{k \in S} P_{i, k} \int_{0}^{t} F_{k, j}(t-u, x-\rho(i) u) \lambda e^{-\lambda u} d u+e^{-\lambda t} 1_{\{h \leq p\}} 1_{\{i=j\}}
$$


Proof. Let $T_{1}$ be the first sojourn time in the initial state. We have

$$
F_{i, j}(t, x)=\int_{0}^{\infty} \mathbb{P}\left\{Y_{t}>x, X_{t}=j \mid T_{1}=u, X_{0}=i\right\} \lambda e^{-\lambda u} d u .
$$

If $u \geq t$ and $X_{0}=i$ we have $Y_{t}=\rho(i) t=r_{p} t$ and $\mathbb{P}\left\{X_{t}=j \mid T_{1}=u, X_{0}=i\right\}=1$ if $i=j$ and 0 otherwise. Moreover, since $r_{p} t>x$ is equivalent to $r_{p} t \geq r_{h} t$, that is $h \leq p$, we obtain

$$
F_{i, j}(t, x)=\int_{0}^{t} \mathbb{P}\left\{Y_{t}>x, X_{t}=j \mid T_{1}=u, X_{0}=i\right\} \lambda e^{-\lambda u} d u+e^{-\lambda t} 1_{\{h \leq p\}} 1_{\{i=j\}} .
$$

Now,

$$
\begin{aligned}
\mathbb{P}\left\{Y_{t}\right. & \left.>x, X_{t}=j \mid T_{1}=u, X_{0}=i\right\} \\
& =\sum_{k \in S} \mathbb{P}\left\{Y_{t}>x, X_{t}=j \mid X_{u}=k, T_{1}=u, X_{0}=i\right\} \mathbb{P}\left\{X_{u}=k \mid T_{1}=u, X_{0}=i\right\} .
\end{aligned}
$$

For the second term in the sum, we have

$$
\begin{aligned}
\mathbb{P}\left\{X_{u}=k \mid T_{1}=u, X_{0}=i\right\} & =\mathbb{P}\left\{X_{T_{1}}=k \mid T_{1}=u, X_{0}=i\right\} \\
& =\mathbb{P}\left\{Z_{1}=k \mid T_{1}=u, Z_{0}=i\right\} \\
& =P_{i, k},
\end{aligned}
$$

For the first one, $T_{1}=u$ and $X_{0}=i$ implies that $Y_{u}=\rho(i) u$, so $\mathbb{P}\left\{Y_{t}>x, X_{t}=j \mid X_{u}=k, T_{1}=u, X_{0}=i\right\}$

$$
\begin{aligned}
& =\mathbb{P}\left\{\int_{u}^{t} \rho\left(X_{v}\right) d v>x-\rho(i) u, X_{t}=j \mid X_{u}=k, T_{1}=u, X_{0}=i\right\} \\
& =\mathbb{P}\left\{\int_{u}^{t} \rho\left(X_{v}\right) d v>x-\rho(i) u, X_{t}=j \mid X_{u}=k\right\} \\
& =\mathbb{P}\left\{Y_{t-u}>x-\rho(i) u, X_{t-u}=j \mid X_{0}=k\right\} \\
& =F_{k, j}(t-u, x-\rho(i) u)
\end{aligned}
$$

where the second equality follows from the Markov property and the third follows from homogeneity. Combining these results, we obtain relation (21).

Corollary 5.5 For $t>0, i, j \in S, 1 \leq h \leq m$ and $x \in\left(r_{h-1} t, r_{h} t\right)$ we have

$$
\frac{\partial F_{i, j}(t, x)}{\partial t}=-\rho(i) \frac{\partial F_{i, j}(t, x)}{\partial x}+\sum_{k \in S} A_{i, k} F_{k, j}(t, x) \text {. }
$$


Proof. Consider equation (21) with $i \in B_{p}, 0 \leq p \leq m$ and $j \in S$.

Differentiating $F_{i, j}(t, x)$ with respect to variable $t$, we get

$$
\begin{aligned}
\frac{\partial F_{i, j}(t, x)}{\partial t}= & \sum_{k \in S} P_{i, k}\left[\int_{0}^{t} \frac{\partial F_{k, j}(t-u, x-\rho(i) u)}{\partial t} \lambda e^{-\lambda u} d u+F_{k, j}(0, x-\rho(i) t) \lambda e^{-\lambda t}\right] \\
& -\lambda e^{-\lambda t} 1_{\{h \leq p\}} 1_{\{i=j\}}
\end{aligned}
$$

Differentiating now $F_{i, j}(t, x)$ with respect to variable $x$, we get

$$
\frac{\partial F_{i, j}(t, x)}{\partial x}=\sum_{k \in S} P_{i, k} \int_{0}^{t} \frac{\partial F_{k, j}(t-u, x-\rho(i) u)}{\partial x} \lambda e^{-\lambda u} d u .
$$

Let us consider the functions $\psi_{k, j}$ and $\varphi_{k, j}$ defined by

$$
\psi_{k, j}(u)=F_{k, j}(t-u, x-\rho(i) u) \quad \text { and } \quad \varphi_{k, j}(u)=\psi_{k, j}(u) e^{-\lambda u} .
$$

Note that we have $\psi_{i, j}(t)=1_{\{h \leq p\}} 1_{\{i=j\}}$, so equation (21) can also be written as

$$
\varphi_{i, j}(0)=\lambda \sum_{k \in S} P_{i, k} \int_{0}^{t} \varphi_{k, j}(u) d u+\varphi_{i, j}(t)
$$

Differentiating $\psi_{k, j}$ with respect to $u$, we get

$$
\psi_{k, j}^{\prime}(u)=-\frac{\partial F_{k, j}(t-u, x-\rho(i) u)}{\partial t}-\rho(i) \frac{\partial F_{k, j}(t-u, x-\rho(i) u)}{\partial x} .
$$

We thus obtain

$$
\begin{aligned}
\frac{\partial F_{i, j}(t, x)}{\partial t}+\rho(i) \frac{\partial F_{i, j}(t, x)}{\partial x} & =-\lambda \sum_{k \in S} P_{i, k} \int_{0}^{t} \psi_{k, j}^{\prime}(u) e^{-\lambda u} d u+\lambda \sum_{k \in S} P_{i, k} \varphi_{k, j}(t)-\lambda \varphi_{i, j}(t) \\
& =\lambda \sum_{k \in S} P_{i, k} \varphi_{k, j}(0)-\lambda^{2} \int_{0}^{t} \varphi_{k, j}(u) d u-\lambda \varphi_{i, j}(t) \\
& =\lambda \sum_{k \in S} P_{i, k} \varphi_{k, j}(0)-\lambda \varphi_{i, j}(0) \\
& =\lambda \sum_{k \in S} P_{i, k} F_{k, j}(t, x)-\lambda F_{i, j}(t, x) \\
& =\sum_{k \in S} A_{i, k} F_{k, j}(t, x)
\end{aligned}
$$

INRIA 
where the second equality is obtained by integration by parts, the third equality follows from relation (23) and the last one from the relation $A=-\lambda(I-P)$.

Let $D$ be the diagonal matrix containing the reward rates $\rho(i)$ and $F(t, x)$ the matrix containing the $F_{i, j}(t, x)$. The forward and backward equations (19) and (22) become respectively in matrix notation

$$
\frac{\partial F(t, x)}{\partial t}=-\frac{\partial F(t, x)}{\partial x} D+F(t, x) A
$$

and

$$
\frac{\partial F(t, x)}{\partial t}=-D \frac{\partial F(t, x)}{\partial x}+A F(t, x)
$$

These equations are hyperbolic partial differential equations having a unique solution on the domain $E$ with the initial condition given by relation (15), see for instance [6]. A method for obtaining these solutions is presented in the following subsection.

\subsection{Solutions}

The solution of the forward partial differential equation (24) is given by the following theorem.

Theorem 5.6 For every $t>0$ and $x \in\left[r_{h-1} t, r_{h} t\right)$ for $h=1,2, \ldots, m$, we have

$$
F(t, x)=\sum_{n=0}^{\infty} e^{-\lambda t} \frac{(\lambda t)^{n}}{n !} \sum_{k=0}^{n}\left(\begin{array}{l}
n \\
k
\end{array}\right) x_{h}^{k}\left(1-x_{h}\right)^{n-k} C^{(h)}(n, k),
$$

where $x_{h}=\frac{x-r_{h-1} t}{\left(r_{h}-r_{h-1}\right) t}$ and the matrices $C^{(h)}(n, k)=\left(C_{B_{u} B_{v}}^{(h)}(n, k)\right)_{0 \leq u, v \leq m}$ are given by the following recursive expressions

for $0 \leq u \leq m$ and $h \leq v \leq m$ :

$$
\begin{aligned}
& \quad \text { for } n \geq 0: \quad C_{B_{u} B_{v}}^{(1)}(n, 0)=\left(P^{n}\right)_{B_{u} B_{v}} \quad \text { and } C_{B_{u} B_{v}}^{(h)}(n, 0)=C_{B_{u} B_{v}}^{(h-1)}(n, n) \text { for } h>1 \\
& \text { for } 1 \leq k \leq n: \\
& C_{B_{u} B_{v}}^{(h)}(n, k)=\frac{r_{v}-r_{h}}{r_{v}-r_{h-1}} C_{B_{u} B_{v}}^{(h)}(n, k-1)+\frac{r_{h}-r_{h-1}}{r_{v}-r_{h-1}} \sum_{w=0}^{m} C_{B_{u} B_{w}}^{(h)}(n-1, k-1) P_{B_{w} B_{v}},
\end{aligned}
$$


for $0 \leq u \leq m$ and $0 \leq v \leq h-1$ :

for $n \geq 0: \quad C_{B_{u} B_{v}}^{(m)}(n, n)=0_{B_{u} B_{v}} \quad$ and $C_{B_{u} B_{v}}^{(h)}(n, n)=C_{B_{u} B_{v}}^{(h+1)}(n, 0)$ for $h<m$

for $0 \leq k \leq n-1$ :

$$
C_{B_{u} B_{v}}^{(h)}(n, k)=\frac{r_{h-1}-r_{v}}{r_{h}-r_{v}} C_{B_{u} B_{v}}^{(h)}(n, k+1)+\frac{r_{h}-r_{h-1}}{r_{h}-r_{v}} \sum_{w=0}^{m} C_{B_{u} B_{w}}^{(h)}(n-1, k) P_{B_{w} B_{v}} .
$$

Proof. For $t>0$ and $x \in\left(r_{h-1} t, r_{h} t\right)$ for $h=1,2, \ldots, m$, we write the solution of the forward equation (24) as

$$
F(t, x)=\sum_{n=0}^{\infty} e^{-\lambda t} \frac{(\lambda t)^{n}}{n !} \sum_{k=0}^{n}\left(\begin{array}{l}
n \\
k
\end{array}\right) x_{h}^{k}\left(1-x_{k}\right)^{n-k} C^{(h)}(n, k),
$$

and we determine the relations that must be satisfied by the matrices $C^{(h)}(n, k)$. We have

$$
\begin{aligned}
\frac{\partial F(t, x)}{\partial t}=-\lambda F(t, x)+\frac{\lambda}{r_{h}-r_{h-1}} \sum_{n=0}^{\infty} e^{-\lambda t} & \frac{(\lambda t)^{n}}{n !} \sum_{k=0}^{n}\left(\begin{array}{l}
n \\
k
\end{array}\right) x_{h}^{k}\left(1-x_{h}\right)^{n-k} \\
& \times\left[r_{h} C^{(h)}(n+1, k)-r_{h-1} C^{(h)}(n+1, k+1)\right],
\end{aligned}
$$

and

$$
\begin{aligned}
\frac{\partial F(t, x)}{\partial x}=\frac{\lambda}{r_{h}-r_{h-1}} \sum_{n=0}^{\infty} e^{-\lambda t} \frac{(\lambda t)^{n}}{n !} \sum_{k=0}^{n}\left(\begin{array}{l}
n \\
k
\end{array}\right) & x_{h}^{k}\left(1-x_{h}\right)^{n-k} \\
& \times\left[C^{(h)}(n+1, k+1)-C^{(h)}(n+1, k)\right] .
\end{aligned}
$$

Since $A=-\lambda(I-P)$, we obtain

$$
F(t, x) A=-\lambda F(t, x)+\lambda F(t, x) P,
$$

that is,

$$
F(t, x) A=-\lambda F(t, x)+\lambda \sum_{n=0}^{\infty} e^{-\lambda t} \frac{(\lambda t)^{n}}{n !} \sum_{k=0}^{n}\left(\begin{array}{l}
n \\
k
\end{array}\right) x_{h}^{k}\left(1-x_{h}\right)^{n-k} C^{(h)}(n, k) P .
$$

It follows that if the matrices $C^{(h)}(n, k)$ are such that

$$
C^{(h)}(n+1, k+1)\left[D-r_{h-1} I\right]=C^{(h)}(n+1, k)\left[D-r_{h} I\right]+\left(r_{h}-r_{h-1}\right) C^{(h)}(n, k) P
$$


then equation (24) is satisfied. The recurrence relation (29) can also be written as follows, for every $h=1, \ldots, m$ and $u=0,1, \ldots, m$

If $v=h, h+1, \ldots, m$ then

$$
C_{B_{u} B_{v}}^{(h)}(n, k)=\frac{r_{v}-r_{h}}{r_{v}-r_{h-1}} C_{B_{u} B_{v}}^{(h)}(n, k-1)+\frac{r_{h}-r_{h-1}}{r_{v}-r_{h-1}} \sum_{w=0}^{m} C_{B_{u} B_{w}}^{(h)}(n-1, k-1) P_{B_{w} B_{v}},
$$

and if $v=0,1, \ldots, h-1$ then

$$
C_{B_{u} B_{v}}^{(h)}(n, k)=\frac{r_{h-1}-r_{v}}{r_{h}-r_{v}} C_{B_{u} B_{v}}^{(h)}(n, k+1)+\frac{r_{h}-r_{h-1}}{r_{h}-r_{v}} \sum_{w=0}^{m} C_{B_{u} B_{w}}^{(h)}(n-1, k) P_{B_{w} B_{v}} .
$$

To get the initial conditions for the $C^{(h)}(n, k)$, we consider the jumps of $F(t, x)$.

We consider first the jump at point $x=r_{0} t=0$. For $t>0$ we have at point $x=0$, that is for $h=1$, from relation (26)

$$
F(t, 0)=\sum_{n=0}^{\infty} e^{-\lambda t} \frac{(\lambda t)^{n}}{n !} C^{(1)}(n, 0),
$$

It follows from relation (15) that for $u, v=0,1, \ldots, m$,

$$
C_{B_{u} B_{v}}^{(1)}(n, 0)=\left(P^{n}\right)_{B_{u} B_{v}}-P_{B_{0} B_{0}}^{n} 1_{\{u=v=0\}} .
$$

This implies in particular that for every $0 \leq u \leq m$

$$
C_{B_{u} B_{v}}^{(1)}(n, 0)=\left(P^{n}\right)_{B_{u} B_{v}} \text { for } 1 \leq v \leq m .
$$

We consider now the jumps at points $x=r_{h} t$ for $1 \leq h \leq m-1$. For $t>0$ and $1 \leq h \leq m-1$ and $i, j \in S$, we have

$$
F_{i, j}\left(t, r_{h} t\right)=\lim _{x \leq r_{h} t} F_{i, j}(t, x)-\mathbb{P}\left\{Y_{t}=r_{h} t, X_{t}=j \mid X_{0}=i\right\} .
$$

From relation (26) and relation (14), we get

$$
C_{B_{u} B_{v}}^{(h+1)}(n, 0)=C_{B_{u} B_{v}}^{(h)}(n, n)+P_{B_{h} B_{h}}^{n} 1_{\{u=v=h\}} .
$$

This implies in particular that for every $0 \leq u \leq m$

$$
C_{B_{u} B_{v}}^{(h)}(n, 0)=C_{B_{u} B_{v}}^{(h-1)}(n, n) \text { for } 1<h \leq v \leq m,
$$

$\mathrm{RR} \mathrm{n}^{\circ} 3806$ 
and

$$
C_{B_{u} B_{v}}^{(h)}(n, n)=C_{B_{u} B_{v}}^{(h+1)}(n, 0) \text { for } 0 \leq v \leq h-1<m-1 .
$$

We consider finally the jump at point $x=r_{m} t$, that is for $h=m$. For $t>0$ we have

$$
0=F_{i, j}\left(t, r_{m} t\right)=\lim _{x \leq r_{m} t} F_{i, j}(t, x)-\mathbb{P}\left\{Y_{t}=r_{h} t, X_{t}=j \mid X_{0}=i\right\},
$$

which leads as in the previous case to the relation

$$
C_{B_{u} B_{v}}^{(m)}(n, n)=P_{B_{m} B_{m}}^{n} 1_{\{u=v=m\}} .
$$

This implies in particular that for every $0 \leq u \leq m$

$$
C_{B_{u} B_{v}}^{(m)}(n, n)=0 \text { for } 0 \leq v \leq m-1 .
$$

The proof is now complete.

Corollary 5.7 For $h=1,2, \ldots, m, n \geq 0$ and $0 \leq k \leq n$, the matrices $C^{(h)}(n, k)=$ $\left(C_{B_{u} B_{v}}^{(h)}(n, k)\right)_{0 \leq u, v \leq m}$ satisfy the following recursive expressions

for $h \leq u \leq m$ and $0 \leq v \leq m$ :

for $n \geq 0: \quad C_{B_{u} B_{v}}^{(1)}(n, 0)=\left(P^{n}\right)_{B_{u} B_{v}} \quad$ and $C_{B_{u} B_{v}}^{(h)}(n, 0)=C_{B_{u} B_{v}}^{(h-1)}(n, n)$ for $h>1$

for $1 \leq k \leq n$ :

$$
C_{B_{u} B_{v}}^{(h)}(n, k)=\frac{r_{u}-r_{h}}{r_{u}-r_{h-1}} C_{B_{u} B_{v}}^{(h)}(n, k-1)+\frac{r_{h}-r_{h-1}}{r_{u}-r_{h-1}} \sum_{w=0}^{m} P_{B_{u} B_{w}} C_{B_{w} B_{v}}^{(h)}(n-1, k-1),
$$

for $0 \leq u \leq h-1$ and $0 \leq v \leq m$ :

for $n \geq 0: \quad C_{B_{u} B_{v}}^{(m)}(n, n)=0_{B_{u} B_{v}} \quad$ and $\quad C_{B_{u} B_{v}}^{(h)}(n, n)=C_{B_{u} B_{v}}^{(h+1)}(n, 0)$ for $h<m$ for $0 \leq k \leq n-1$ :

$$
C_{B_{u} B_{v}}^{(h)}(n, k)=\frac{r_{h-1}-r_{u}}{r_{h}-r_{u}} C_{B_{u} B_{v}}^{(h)}(n, k+1)+\frac{r_{h}-r_{h-1}}{r_{h}-r_{u}} \sum_{w=0}^{m} P_{B_{u} B_{w}} C_{B_{w} B_{v}}^{(h)}(n-1, k) .
$$

INRIA 
Proof. The proof is the same as the proof of theorem 5.6 using the backward equation (25) and the expression (26). We thus obtain that the matrices $C^{(h)}(n, k)$ satisfy the relation

$$
\left[D-r_{h-1} I\right] C^{(h)}(n+1, k+1)=\left[D-r_{h} I\right] C^{(h)}(n+1, k)+\left(r_{h}-r_{h-1}\right) P C^{(h)}(n, k) .
$$

The recurrence relation (33) can also be written as follows, for every $h=1, \ldots, m$ and $v=0,1, \ldots, m$

If $u=h, h+1, \ldots, m$ then

$$
C_{B_{u} B_{v}}^{(h)}(n, k)=\frac{r_{u}-r_{h}}{r_{u}-r_{h-1}} C_{B_{u} B_{v}}^{(h)}(n, k-1)+\frac{r_{h}-r_{h-1}}{r_{u}-r_{h-1}} \sum_{w=0}^{m} P_{B_{u} B_{w}} C_{B_{w} B_{v}}^{(h)}(n-1, k-1),
$$

and if $u=0,1, \ldots, h-1$ then

$$
C_{B_{u} B_{v}}^{(h)}(n, k)=\frac{r_{h-1}-r_{u}}{r_{h}-r_{u}} C_{B_{u} B_{v}}^{(h)}(n, k+1)+\frac{r_{h}-r_{h-1}}{r_{h}-r_{u}} \sum_{w=0}^{m} P_{B_{u} B_{w}} C_{B_{w} B_{v}}^{(h)}(n-1, k) .
$$

As for the proof of theorem 5.6, we consider the jumps of $F(t, x)$.

Relation (30) implies that for every $0 \leq v \leq m$

$$
C_{B_{u} B_{v}}^{(1)}(n, 0)=\left(P^{n}\right)_{B_{u} B_{v}} \text { for } 1 \leq u \leq m .
$$

Relation (31) implies that for every $0 \leq v \leq m$

$$
C_{B_{u} B_{v}}^{(h)}(n, 0)=C_{B_{u} B_{v}}^{(h-1)}(n, n) \text { for } 1<h \leq u \leq m
$$

and

$$
C_{B_{u} B_{v}}^{(h)}(n, n)=C_{B_{u} B_{v}}^{(h+1)}(n, 0) \text { for } 0 \leq u \leq h-1<m-1 .
$$

Finally, relation (32) implies that for every $0 \leq v \leq m$

$$
C_{B_{u} B_{v}}^{(m)}(n, n)=0 \text { for } 0 \leq u \leq m-1 .
$$

This completes the proof.

The following corollary gives an upper bound for the matrices $C^{(h)}(n, k)$. If $M$ and $K$ are two square matrices with the same dimension, the notation $M \leq K$ means that for every $i, j$, we have $M_{i, j} \leq K_{i, j}$. 
Corollary 5.8 For every $n \geq 0,0 \leq k \leq n$ and $1 \leq h \leq m$, we have

$$
0 \leq C^{(h)}(n, k) \leq P^{n}
$$

Proof. The proof is easily made by a two level induction; first over the integer $n$ and then, for fixed $n$, over the integer $k$, using the recurrence relation described in theorem 5.6 or equivalently in corollary 5.7. The result is evidently true for $n=0$. Note that in relation (27), that is for $h \leq v$, we have

$$
0 \leq \frac{r_{v}-r_{h}}{r_{v}-r_{h-1}}=1-\frac{r_{h}-r_{h-1}}{r_{v}-r_{h-1}} \leq 1,
$$

and in relation (28), that is for $v \leq h-1$, we have

$$
0 \leq \frac{r_{h-1}-r_{v}}{r_{h}-r_{v}}=1-\frac{r_{h}-r_{h-1}}{r_{h}-r_{v}} \leq 1 .
$$

Consider first the case $v \leq h-1$. The result is true for the couple $(n, n)$, since in this case we have $C_{B_{u} B_{v}}^{(m)}(n, n)=0$. Suppose the result is true for integer $n-1$ and for the couple $(n, k+1)$, then using relation (28), we get $C_{B_{u} B_{v}}^{(h)}(n, k) \geq 0$ and

$$
\begin{aligned}
C_{B_{u} B_{v}}^{(h)}(n, k) & =\frac{r_{h-1}-r_{v}}{r_{h}-r_{v}} C_{B_{u} B_{v}}^{(h)}(n, k+1)+\frac{r_{h}-r_{h-1}}{r_{h}-r_{v}} \sum_{w=0}^{m} C_{B_{u} B_{w}}^{(h)}(n-1, k) P_{B_{w} B_{v}} \\
& \leq \frac{r_{h-1}-r_{v}}{r_{h}-r_{v}}\left(P^{n}\right)_{B_{u} B_{v}}+\frac{r_{h}-r_{h-1}}{r_{h}-r_{v}} \sum_{w=0}^{m}\left(P^{n-1}\right)_{B_{u} B_{w}} P_{B_{w} B_{v}} \\
& =\frac{r_{h-1}-r_{v}}{r_{h}-r_{v}}\left(P^{n}\right)_{B_{u} B_{v}}+\frac{r_{h}-r_{h-1}}{r_{h}-r_{v}}\left(P^{n}\right)_{B_{u} B_{v}} \\
& =\left(P^{n}\right)_{B_{u} B_{v}} .
\end{aligned}
$$

The same argument is used in the case where $h \leq v$ from relation (27). Moreover, the relation

$$
C_{B_{u} B_{v}}^{(h)}(n, 0)=C_{B_{u} B_{v}}^{(h-1)}(n, n) \text { for } 1<h \leq v \leq m,
$$

and

$$
C_{B_{u} B_{v}}^{(h)}(n, n)=C_{B_{u} B_{v}}^{(h+1)}(n, 0) \text { for } 0 \leq v \leq h-1<m-1,
$$

are used in the recurrence to take into account the evolution of the integer $h$ in both cases $v \leq h-1$ and $h \leq v$.

This result is particularly interesting form a computational point of view to avoid overflow problems. 


\section{References}

[1] H. A. David. Order statistics. John Wiley \& sons, Inc., New York - London - Sidney - Toronto, 1981.

[2] E. de Souza e Silva and H. R. Gail. Calculating cumulative operational time distributions of repairable computer systems. IEEE Trans. Computers, 35, april 1986.

[3] E. de Souza e Silva and H. R. Gail. An algorithm to calculate transient distributions of cumulative rate and impulse based reward. Communications in Statistics - Stochastic Models, 14(3), 1998.

[4] S. Karlin and H. W. Taylor. A second course in stochastic processes. Academic Press, New York - San Francisco - London, 1981.

[5] H. Nabli and B. Sericola. Performability analysis: A new algorithm. IEEE Trans. Computers, 45(4), april 1996.

[6] I. G. Petrovsky. Lectures on partial differential equations. Interscience publishers, New York, 1962.

[7] S.M. Ross. Stochastic Processes. John Wiley and Sons, 1983.

[8] G. Rubino and B. Sericola. Interval availability distribution computation. In 23th IEEE International Symposium on Fault Tolerant Computing (FTCS'23), Toulouse, France, june 1993.

[9] G. Rubino and B. Sericola. Interval availability analysis using denumerable Markov processes. Application to multiprocessor subject to breakdowns and repair. IEEE Trans. Computers. Special Issue on Fault-Tolerant Computing, 44(2), february 1995.

[10] B. Sericola. Closed-form solution for the distribution of the total time spent in a subset of states of a homogeneous markov process during a finite observation period. J. Appl. Prob., 27, 1990.

RR $\mathrm{n}^{\circ} 3806$ 


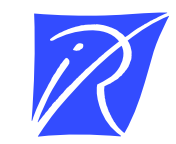

Unité de recherche INRIA Lorraine, Technopôle de Nancy-Brabois, Campus scientifique, 615 rue du Jardin Botanique, BP 101, 54600 VILLERS LÈS NANCY

Unité de recherche INRIA Rennes, Irisa, Campus universitaire de Beaulieu, 35042 RENNES Cedex

Unité de recherche INRIA Rhône-Alpes, 655, avenue de l'Europe, 38330 MONTBONNOT ST MARTIN

Unité de recherche INRIA Rocquencourt, Domaine de Voluceau, Rocquencourt, BP 105, 78153 LE CHESNAY Cedex

Unité de recherche INRIA Sophia-Antipolis, 2004 route des Lucioles, BP 93, 06902 SOPHIA-ANTIPOLIS Cedex

Éditeur

INRIA, Domaine de Voluceau, Rocquencourt, BP 105, 78153 LE CHESNAY Cedex (France)

http://www.inria.fr

ISSN 0249-6399 